KŌDAI MATH. SEM. REP.

21 (1969), 16-45

\title{
HOMOGENEOUS CONTACT MANIFOLDS AND ALMOST FINSLER MANIFOLDS
}

\author{
By Kentaro Yano And Yosio Mutō
}

\section{§1. Introduction.}

The study of geometrical properties of spaces admitting homogeneous contact transformations was started by Doyle [6], Eisenhart [7, 8], Hosokawa [11], Knebelman [8], Mutō [13,14] and Yano [14, 22]. Eisenhart and Knebelman [8] introduced the notion of contact frames which are now called the first contact frames, and Doyle [6] and Muto [13] introduced independently the notion of the second contact frames. The theory of contact frames was developed in a more systematic way by Yano and Davies [20] on the basis of the theory of distributions [17], [18].

On the other hand Boothby and Wang [1] and Gray [9] threw light upon geometrical properties of spaces admitting contact transformations by introducing the notion of contact structures. Guided by this notion and the paper of Yano and Davies [20] the present authors studied a manifold which admits a structure called the homogeneous contact structure and at the same time an almost product structure and showed that these structures induce contact frames [22].

There are some close relations between spaces with contact frames and Finsler spaces as shown by Mutō and Yano [14] and Yano and Davies [20]. The main purpose of the present paper is to study these relations in a more systematic way using the notions of homogeneous contact structure and of almost product structure.

An analytical definition of homogeneous contact transformations is the following [7].

Let $x^{h}$ and $p_{i}(h, i, j, \cdots=1, \cdots, n)$ be $2 n$ independent variables and $\bar{x}^{h}, \bar{p}_{i}$ another set of $2 n$ variables. A change of variables from $(x, p)$ to $(\bar{x}, \bar{p})$, where

$$
\begin{aligned}
& \bar{x}^{n}=\bar{x}^{n}\left(x^{1}, \cdots, x^{n} ; p_{1}, \cdots, p_{n}\right), \\
& \bar{p}_{i}=\bar{p}_{i}\left(x^{1}, \cdots, x^{n} ; p_{1}, \cdots, p_{n}\right)
\end{aligned}
$$

are $2 n$ differentiable functions, is called a homogeneous contact transformation if we have $\bar{p}_{i} d \bar{x}^{i}=p_{i} d x^{2}$ and the Jacobian does not vanish. In this definition we assume that the point $\left(x^{1}, \cdots, x^{n} ; p_{1}, \cdots, p_{n}\right)$ is in some suitable domain of the $2 n$ dimensional number space.

Geometrically, one can interprete homogeneous contact transformations in two ways.

One of these is the following [16].

A homogeneous contact transformation is a transformation on the cotangent

Received May 9, 1968. 
bundle $T^{*}(M)$ over an $n$-dimensional differentiable manifold $M$ which is not bundle preserving. Let $\mathrm{P}$ be a point of $M$. If we take a coordinate neighborhood of $\mathrm{P}, \mathrm{P}$ is denoted by local coordinates $\xi^{h}(h, i, j, \cdots=1, \cdots, n)$. An element of $T_{\mathrm{P}}^{*}$ is denoted by $\xi^{h^{*}}\left(h^{*}, i^{*}, j^{*}, \cdots=n+1, \cdots, 2 n\right)$ in the natural frame. Let $H$ be a transformation which takes an element $A$ of $T^{*}(M)$ into an element $\bar{A}$ of $T^{*}(M)$. Let $\pi$ be the projection of the cotangent bundle: $T^{*}(M) \rightarrow M$. If we take coordinate neighborhoods $U$ and $V$ respectively of $\pi A$ and $\pi H A, A$ and $H A$ are denoted by $\left(\xi^{1}, \cdots, \xi^{n} ; \xi^{1^{*}}, \cdots, \xi^{n^{*}}\right)$ and $\left(\bar{\xi}^{1}, \cdots, \bar{\xi}^{n} ; \bar{\xi}^{1^{*}}, \cdots, \bar{\xi}^{n *}\right)$ respectively. $H$ is called a homogeneous contact transformation if $H$ is a diffeomorphic transformation of $T^{*}(M)$ and the functions $\bar{\xi}^{n}\left(\xi^{1}, \cdots, \xi^{n} ; \xi^{1^{*}}, \cdots, \xi^{n^{*}}\right), \bar{\xi}^{h^{*}}\left(\xi^{1}, \cdots, \xi^{n} ; \xi^{1^{*}}, \cdots, \xi^{n^{*}}\right)$ satisfy $\bar{\xi}^{i^{*}} d \bar{\xi}^{i}=\xi^{i^{*}} d \xi^{i}$. $H$ does not in general preserve the bundle structure of $T^{*}(M)$.

The other way of interpreting homogeneous contact transformations is the following. We consider a $2 n$-dimensional differentiable manifold which is covered by a set of coordinate neighborhoods in such a way that for any two coordinate neighborhoods $U, U^{\prime}$ satisfying the condition $U \cap U^{\prime} \neq \phi$ the transformation of coordinates $\left(\xi^{1}, \cdots, \xi^{n} ; \xi^{1^{*}}, \cdots, \xi^{n *}\right) \rightarrow\left(\xi^{1^{\prime}}, \cdots, \xi^{n^{\prime}} ; \xi^{1^{* \prime}}, \cdots, \xi^{n * \prime}\right)$ satisfies $\xi^{i^{*}} d \xi^{i}=\xi^{i^{* \prime}} d \xi^{i^{\prime}}$. In this interpretation a homogeneous contact transformation appears as a coordinate transformation.

Homogeneous contact transformations are intimately related to a set of transformations called contact transformations. A geometrical interpretation of contact transformations was done by Boothby and Wang [1] and Gray [9] and the notion of contact structure was introduced. Hence it is natural to take homogeneous contact transformations as coordinate transformations and introduce an object which might be called a homogeneous contact structure. This was done in [20]. A homogeneous contact structure is a global 1-form $\omega$ such that $\omega \neq 0$ and $(d \omega)^{n} \neq 0$ throughout the given $2 n$-dimensional manifold $M$. In [20] the notion of contact frames is also discussed. Some of the results obtained in [20] which we use in the present paper are summarized in $\S 2$.

A Finsler space $[4,15]$ is a differentiable manifold $M$ of dimension $n$ in which the length of a smooth curve is given by

$$
s=\int_{t_{0}}^{t} F\left(x^{1}, \cdots, x^{n} ; y^{1}, \cdots, y^{n}\right) d t, \quad y^{h}=\frac{d x^{h}}{d t},
$$

where $F(x, y)$ is a homogeneous function of degree one with respect to $y^{1}, \cdots, y^{n}$. $F$ must satisfy some additional conditions.

Fruitful studies of Finsler spaces from a point of view of geometry of connections have been made since the notion of elements of support was introduced by Cartan [4]. An element of support is a set $(\mathrm{P}, Y)$ where $\mathrm{P}$ is a point of $M$ and $Y$ an element of $T_{\mathrm{P}}(M)$. Thus a $(p, q)$-tensor field of a Finsler space is a differentiable map $\varphi$ of $T(M)$ into the $(p, q)$-tensor bundle $T_{q}^{p}(M)$ over $M$ such that $\pi(\varphi(\mathrm{P}, Y))=\mathrm{P}$ and $\varphi(\mathrm{P}, \lambda Y)=\lambda^{k} \varphi(\mathrm{P}, Y)$ where $\pi$ is the projection $T(M) \rightarrow M$ and $k$ is an integer. This implies that a Finsler space whose base manifold is $M$ is the bundle space $T(M)$ in which some special structure is assumed.

A necessary and sufficient condition for the tangent bundle $T(M)$ endowed with a structure $F$ to be a Finsler space in the sense of Cartan $[4,15]$ was 
obtained by Yano and Davies [21].

Let $T_{\mathrm{P}}^{*}(M)$ be the dual space of $T_{\mathrm{P}}(M) . \quad T^{*}(M)=\cup_{\mathrm{P}} T_{\mathrm{P}}^{*}(M)$ is the cotangent bundle. The structure in $T^{*}(M)$ which is induced from $F$ is denoted by $F^{*}$. Assuming the structure $F^{*}$ in $T^{*}(M)$ we get a space which is almost the same as a Cartan space [3]. Since we can always return to the original tangent bundle with the structure $F$ and the correspondence between $T(M)$ with $F$ and $T^{*}(M)$ with $F^{*}$ is such that the structure of the one determines that of the other, we can call the space $T^{*}(M)$ with $F^{*}$ a Finsler space in the extended sense.

Now let us turn our attention to homogeneous contact manifolds, namely, manifolds with homogeneous contact structure. We consider a homogeneous contact manifold with two complementary distributions and introduce a metric tensor and an affine connection. Since this affine connection is not the Riemannian connection induced by the metric tensor, we rather call the tensor an almost metric tensor. If such structures satisfy a set of conditions we say that the manifold is an almost Finsler manifold. This manifold is characterized by the fact that it becomes a Finsler manifold if one distribution- $p_{B}{ }^{A} d \xi^{B}=0$ - is completely integrable.

\section{§2. Even-dimensional contact manifold.}

Let $M$ be a $2 n$-dimensional connected manifold of class $C^{\infty}$. It is called an even-dimensional contact manifold if there exists a global differentiable one-form $\omega$ satisfying

$$
\omega \neq 0, \quad(d \omega)^{n} \neq 0
$$

throughout the manifold $M$, where the expression $(d \omega)^{n}$ means the exterior product $d \omega \wedge \cdots \wedge d \omega$ with $n$ factors. The one-form $\omega$ is called a homogeneous contact structure.

Let $M$ be an even-dimensional contact manifold. Taking an open covering $\left\{U_{\lambda} ; \lambda \in \Lambda\right\}$ of $M$ where $U_{\lambda}$ is a coordinate neighborhood with local coordinates $\xi^{A}$ $(A=1, \cdots, 2 n)$, we can write

$$
\omega=y_{A} d \xi^{A}
$$

Then we get $\operatorname{det}\left(f_{B A}\right) \neq 0$, where

$$
f_{B A}=\partial_{B} y_{A}-\partial_{A} y_{B} \quad\left(\partial_{B}=\partial / \partial \xi^{B}\right) .
$$

According to Cartan $[2,5]$ there exists a covering by local coordinate systems such that $\omega$ can be written in the form

$$
\omega=\xi^{1^{*}} d \xi^{1}+\cdots+\xi^{n *} d \xi^{n}
$$

hence $^{1)}$

1) Indices run as follows: $A, B, C, D=1, \cdots, 2 n ; h, i, j, k=1, \cdots, n ; h^{*}, i^{*}, j^{*}, k^{*}=1^{*}, \cdots, n^{*}$ $=n+1, \ldots, 2 n$. We use summation convention in the usual way and moreover in the following way:

$$
\sum i \xi^{n+\imath} d \xi^{\imath}=\xi^{i^{*}} d \xi^{\imath}
$$




$$
\omega=\xi^{i^{*}} d \xi^{i} .
$$

This admits us to put

$$
y_{i}=\xi^{i^{*}}, \quad y_{i^{*}}=0 .
$$

Such local coordinates $\xi^{1}, \cdots, \xi^{n} ; \xi^{1^{*}}, \cdots, \xi^{n^{*}}$ will be called contact coordinates. We use only such coordinates in the sequel. Thus we have

$$
f_{j i}=0, \quad f_{j * i}=\delta_{i}^{j}, \quad f_{j i *}=-\delta_{j}^{i}, \quad f_{j * i *}=0 .
$$

Let $U$ and $U^{\prime}$ be coordinate neighborhoods and $\xi^{A}$ and $\xi^{A^{\prime}}$ be the corresponding contact coordinates respectively. If $U \cap U^{\prime}$ is non-empty, we have a coordinate transformation between $\xi^{A}$ and $\xi^{A^{\prime}}$, which must satisfy

$$
\xi^{i^{*}} d \xi^{i}=\xi^{i^{* \prime}} d \xi^{i^{\prime}} .
$$

Thus the coordinate transformations are homogeneous contact transformations.

An even-dimensional contact manifold $M$ is said to have a contact almost product structure if there exist in $M$ globally two differentiable simple $n$-forms $\tilde{\omega}$ and $\tilde{\omega}^{*}$ which are defined only up to non-vanishing scalar multiples and have the following properties. $\tilde{\boldsymbol{\omega}}$ and $\tilde{\boldsymbol{\omega}}^{*}$ satisfy

$$
\tilde{\omega}^{*} \wedge \tilde{\omega} \neq 0, \quad \tilde{\omega}^{*} \wedge \omega \neq 0
$$

and, when $\tilde{\omega}^{*}$ is expressed locally in the form

$$
\rho \tilde{\omega}^{*}=\omega^{1^{*}} \wedge \cdots \wedge \omega^{n *},
$$

where $\omega^{i^{*}}$ are $n$ differentiable one-forms, then there exist locally $n$ differentiable one-forms $\omega^{2}$ satisfying

$$
\sigma \tilde{\omega}=\omega^{1} \wedge \cdots \wedge \omega^{n}
$$

and

$$
d \omega=\omega^{i^{*}} \wedge \omega^{2} .
$$

The main result of our previous paper [22] can be stated in the following form.

Theorem A. Let $M$ be an even-dimensional contact manifold with a contact almost product structure $\left(\tilde{\omega}, \tilde{\omega}^{*}\right)$. Then $M$ has an open covering $\left\{U_{\lambda} ; \lambda \in \Lambda\right\}$ such that in each coordinate neighborhood $U_{\lambda}$ there exist some local bases $\omega^{2}, \omega^{i^{*}}$ of $\tilde{\omega}, \tilde{\omega}^{*}$ having the form

$$
\begin{array}{cl}
\omega^{i}=d \xi^{i}+\Pi^{i h} \omega^{h^{*}} & \Pi^{i h}=\Pi^{h i}, \\
\omega^{i^{*}}=d \xi^{*}-\Gamma_{i h} d \xi^{h} & \Gamma_{i h}=\Gamma_{h \imath} .
\end{array}
$$

$\Gamma_{i n}$ is the first contact frame and $\Pi^{i h}$ is the second contact frame.

In the sequel we use only local bases $\omega^{2}, \omega^{i^{*}}$ and contact coordinates $\xi^{A}$ satisfying (2.7). Such local coordinates are called canonical contact coordinates.

The structure $\left(\tilde{\omega}, \tilde{\omega}^{*}\right)$ is equivalent to a pair of distributions $\mathscr{Q}, Q$, where $\mathscr{Q}$ is determined by $\omega^{i^{*}}=0$ and $Q$ is determined by $\omega^{\imath}=0$. 
Let $P$ be a $(1,1)$-tensor field defined by

$$
\begin{aligned}
p_{i}{ }^{h} & =\delta_{i}^{h}-\Gamma_{i k} \Pi^{k h}, & & p_{i}^{h^{*}}=\left(\delta_{i}^{k}-\Gamma_{i l} I I l k\right) \Gamma_{k h}, \\
p_{i *}{ }^{h} & =\Pi^{i h}, & p_{i^{*}}{ }^{*} & =\Pi^{i k} \Gamma_{k h},
\end{aligned}
$$

and let $Q$ be the tensor field $Q=E-P$ where $E$ is the unit $(1,1)$-tensor field. Then $P$ and $Q$ are projection operators satisfying

$$
\begin{aligned}
& P+Q=I, \quad P^{2}=P, \quad Q^{2}=Q, \quad P Q=Q P=0, \\
& H P=P H=P, \quad H Q=Q H=-Q, \quad H^{2}=I,
\end{aligned}
$$

where $I$ is the identity operator and $H$ is defined by $H=P-Q . \quad H$ is an almost product structure. The components $q_{B}{ }^{A}$ of $Q$ are given by

$$
\begin{aligned}
& q_{i}^{h}=\Gamma_{i k} l I^{k h}, \quad q_{i}^{h^{*}}=-\Gamma_{i h}+\Gamma_{i l} \Pi^{l k} \Gamma_{k h}, \\
& q_{i *^{h}}=-\Pi^{i \hbar}, \quad q_{i *^{*^{*}}}=\delta_{h}^{2}-\Pi^{i k} \Gamma_{k h} .
\end{aligned}
$$

Any vector field of $M$ is projected into $\mathscr{L}$ by $P$ and into $Q$ by $Q$.

\section{§3. Holonomic components and non-holonomic components.}

Hitherto we used only natural frames attached to coordinate systems to represent a tensor by its components. Since we have projection operators, we can derive from a natural frame $\left\{\partial_{A}\right\}$ a frame $\left\{D_{A}\right\}$ which fits to the distributions $\mathscr{Q}$ and $Q$. This frame is composed of the vectors

$$
\begin{aligned}
D_{i} & =\partial_{i}+\Gamma_{i h} \partial_{h^{*},} \\
D_{i^{*}} & =\partial_{i^{*}}-\Pi^{i n} D_{h},
\end{aligned}
$$

hence we have

$$
D_{A}=Y_{A}^{B} \partial_{B}, \quad \partial_{A}=Z_{A}^{B} D_{B}
$$

if we put

$$
\begin{aligned}
Y_{i}^{h} & =\delta_{i}^{h}, & Y_{i}^{h^{*}} & =\Gamma_{i h}, \\
Y_{i^{*}}{ }^{h} & =-\Pi^{i h}, & Y_{i^{h^{*}}} & =\delta_{h}^{i}-\Pi^{i k} \Gamma_{k h}, \\
Z_{i}^{h} & =\delta_{i}^{h}-\Gamma_{i k} \Pi^{k h}, & Z_{i}^{h^{*}} & =-\Gamma_{i h}, \\
Z_{i^{*}}{ }^{h} & =\Pi^{i h}, & Z_{i^{*}}{ }^{*} & =\delta_{h .}^{i} .
\end{aligned}
$$

If a contravariant vector has components $u^{A}$ and $U^{A}$ with respect to the frames $\left\{\partial_{A}\right\}$ and $\left\{D_{A}\right\}$ respectively, then we have

$$
U^{A}=Z_{B}{ }^{A} u^{B}, \quad u^{A}=Y_{B}{ }^{A} U^{B} .
$$

$u^{A}$ and $U^{A}$ will be called respectively the holonomic components and the nonholonomic components of the vector under consideration. Similarly, we say that 
a tensor, for example, a $(1,1)$-tensor has holonomic components $t_{B}{ }^{A}$ and nonholonomic components $T_{B}{ }^{A}$ if

$$
T_{B}^{A}=Y_{B}^{D} Z_{C}{ }^{A} t_{D}^{C}, \quad t_{B}^{A}=Z_{B}^{D} Y_{C}^{A} T_{D}^{C} .
$$

In the sequel the kernel letters of holonomic components will be small letters and the kernel letters of non-holonomic components will be capital letters.

The non-holonomic components $P_{B}{ }^{A}, Q_{B}{ }^{A}$ of the projection tensors $P, Q$ are of the form

$$
P_{B}{ }^{A}=\left[\begin{array}{cc}
\delta_{i}^{h} & 0 \\
0 & 0
\end{array}\right], \quad Q_{B}{ }^{A}=\left[\begin{array}{rr}
0 & 0 \\
0 & \delta_{i^{*}}^{h^{*}}
\end{array}\right] .
$$

Hence a vector field $l$ whose components $L^{h^{*}}$ satisfy $L^{h^{*}}=0$ belongs to the distribution $\mathscr{Q}$ and a vector field $m$ whose components $M^{h}$ satisfy $M^{h}=0$ belongs to the distribution $Q$.

The non-holonomic components $F_{B A}$ of the fundamental 2 -form $d \omega$ satisfy

$$
F_{i h}=F_{i * h^{*}}=0, \quad F_{i * h}=-F_{i h^{*}}=\delta_{i h},
$$

hence we have $f_{B A}=F_{B A}$.

$\S 4$. Almost metric tensor and almost complex structure in an even-dimensional contact manifold.

Let us quote here the following proposition proved by Hatakeyama [10] and Lichnerowicz [12] independently.

Proposition 4.1. Let $M$ be a 2n-dimensional differentiable manifold which admits globally a skew symmetric tensor field $\varphi_{B A}$ whose rank is $2 n$ at every point of $M$. Then $M$ admits globally a pair of symmetric tensor fields $g_{B A}, g^{B A}$ satisfying $g_{B C} g^{C A}=\delta_{B}^{A}$ and such that $\varphi_{B C} g^{C A}$ is an almost complex structure.

In our case the even-dimensional contact manifold $M$ admits the skew-symmetric tensor field $f_{B A}$ whose components are given by (2.6). Thus $M$ admits a symmetric tensor field $g^{B A}$ such that $\operatorname{det}\left(g^{B A}\right) \neq 0$ and

$$
f_{B L} g^{L C} f_{C K} g^{K A}=-\delta_{B}^{A} .
$$

(4. 1) is equivalent to

$$
\begin{aligned}
& g^{i * k} g^{k^{*} h}-g^{i * k^{*}} g^{k h}=-\delta_{i}^{h} \\
& g^{i k} g^{k^{*} h}-g^{i k^{*}} g^{k h}=0 \\
& g^{i * k} g^{k^{*} h^{*}}-g^{i * k^{*}} g^{k h^{*}}=0 .
\end{aligned}
$$

On the other hand the system of equations $g_{B C} g^{C A}=\delta_{B}^{A}$ is equivalent to 


$$
\begin{aligned}
& g_{i k} g^{k h}+g_{i k} g^{k^{*} h}=\delta_{i}^{h}, \\
& g_{i^{*} k} g^{k h}+g_{i^{*} k} g^{k^{*} h}=0, \\
& g_{i k} g^{k h^{*}}+g_{i k^{*}} g^{k^{*} h^{*}}=0, \\
& g_{i^{*} k} g^{k h^{*}}+g_{i^{*} *} g^{k^{*} h^{*}}=\delta_{h^{2}}^{i} .
\end{aligned}
$$

From the two sets of equations written above we get

$$
\begin{aligned}
& g_{i h}=g^{i * h^{*}}, \quad g_{i h^{*}}=-g^{i * h}, \\
& g_{i * h}=-g^{i h^{*}}, \quad g_{i^{*} h^{*}}=g^{i h} .
\end{aligned}
$$

Let us call a differentiable symmetric tensor field $g_{B A}$ such that $\operatorname{det}\left(g_{B A}\right) \neq 0$ an almost metric tensor field. Then we have

Proposition 4.2. Let $M$ be an even-dimensional contact manifold with homogeneous contact structure $\omega$ and let $f_{B A}$ be defined by

$$
d \omega=\frac{1}{2} f_{B A} d \xi^{B} \wedge d \xi^{A} .
$$

Then a necessary and sufficient condition for $f_{B C} g^{C A}=f_{B}{ }^{A}$ to be an almost complex structure is that the almost metric tensor $g_{B A}$ satisfy

$$
\begin{aligned}
& g_{i l} g_{l^{*} h^{*}}-g_{i l^{*}} g_{l h^{*}}=\delta_{i}^{h}, \\
& g_{i^{*} l} g_{l^{*} h^{*}}-g_{i^{*} l^{*}} g_{l^{*}}=0, \\
& g_{i l} g_{l^{*} h}-g_{i l^{*}} g_{l h}=0
\end{aligned}
$$

in a contact coordinate system and $g^{B A}$ is defined by $g_{B C} g^{C A}=\delta_{B}^{A}$. Then $g^{B A}$ satisfies (4. 1$)$ and the structure $\left(f_{B}{ }^{A}, g_{B A}\right)$ is an almost Hermitian structure if $g_{B A}$ defines a Riemannian metric.

It is well known that a homogeneous contact transformation $\xi^{A} \rightarrow \xi^{A^{\prime}}$ satisfies the equations $[7,8,20]$

$$
\xi^{\xi^{*}} \frac{\partial \xi^{h^{\prime}}}{\partial \xi^{i^{*}}}=0, \quad \xi^{i^{*}} \frac{\partial \xi^{h^{* \prime}}}{\partial \xi^{i^{*}}}=\xi^{h^{* \prime}}
$$

This shows that $\xi^{h^{\prime}}$ and $\xi^{h^{* \prime}}$ are homogeneous functions in $\xi^{*}$, namely, in $\xi^{1^{*}}, \cdots, \xi^{n^{*}}$, of degree 0 and 1 respectively. This also proves that a contravariant vector whose components are $\left(0, \cdots, 0 ; \xi^{1^{*}}, \cdots, \xi^{n *}\right)$ in the coordinates $\left\{\xi^{A}\right\}$ has components $\left(0, \cdots, 0 ; \xi^{1^{* \prime}}, \cdots, \xi^{n *^{\prime \prime}}\right)$ in the coordinates $\left\{\xi^{A^{\prime}}\right\}$.

Definition. We call the vector field $p^{A}$ defined by

$$
p^{h}=0, \quad p^{h^{*}}=\xi^{h^{*}}
$$

the natural vector field of $M$.

Let us assume that the Lie derivative of the projection tensor $p_{B}{ }^{A}$ with respect to the natural vector field $p^{A}$ vanishes, that is, 


$$
p^{C} \partial_{C} p_{B}{ }^{A}-p_{B}{ }^{C} \partial_{C} p^{A}+p_{C}{ }^{A} \partial_{B} p^{C}=0 .
$$

From (2.8) we immediately find that $\Pi^{i h}, \Gamma_{i k} \Pi^{k h}$ and $\left(\delta_{i}^{k}-\Gamma_{i l} I^{i k}\right) \Gamma_{k h}$ are homogeneous functions of degree $-1,0$ and 1 respectively of $\xi^{*}$. If we use the symbol

$$
d^{*} f \stackrel{\text { def }}{=} \xi^{i^{*}} \partial_{i^{*}} f
$$

we immediately get

$$
\begin{aligned}
& d^{*} \Pi^{i h}+\Pi^{i h}=0, \\
& \left(d^{*} \Gamma_{i k}-\Gamma_{i k}\right) \Pi^{k h}=0, \\
& \left(\delta_{i}^{k}-\Gamma_{i l} \Pi^{l k}\right)\left(d^{*} \Gamma_{k h}-\Gamma_{k h}\right)=0,
\end{aligned}
$$

from which we can easily deduce that $\Gamma_{i n}$ are homogeneous of degree 1 in $\xi^{*}$.

We further assume that any tensor field which appears in our manifold bearing some geometrical meaning must be such that its Lie derivative with respect to the natural vector field $p^{A}$ is equal to some multiple of the tensor field itself. For example, if $t_{C B}{ }^{A}$ is a tensor field, it must satisfy

$$
p^{D} \partial_{D} t_{C B}{ }^{A}-t_{C B}{ }^{D} \partial_{D} p^{A}+t_{D B}{ }^{A} \partial_{C} p^{D}+t_{C D}{ }^{A} \partial_{B} p^{D}=r t_{C B}{ }^{A} .
$$

Thus we get

$$
\begin{aligned}
& d^{*} t_{j i}{ }^{h}=r t_{j i}{ }^{h}, \quad \quad d^{*} t_{j i}{ }^{h^{*}}=(r+1) t_{j i}{ }^{h^{*}}, \\
& d^{*} t_{j * i}{ }^{h}=(r-1) t_{j * i}{ }^{h}, \quad d^{*} t_{j * i} h^{*}=r t_{j * i} h^{*}, \\
& d^{*} t_{j i^{*}}{ }^{h}=(r-1) t_{j i^{*}}{ }^{h}, \quad d^{*} t_{j i *^{*}}{ }^{* *}=r t_{j i^{*}}{ }^{n^{*}}, \\
& d^{*} t_{j * i *}{ }^{h}=(r-2) t_{j * i i^{*}}{ }^{h}, \quad d^{*} t_{j * i *}{ }^{h^{*}}=(r-1) t_{j * v^{*}}{ }^{l^{*}}
\end{aligned}
$$

and this shows that the components $t_{j i}{ }^{h}$ are homogeneous of degree $r$, the components $t_{j i}{ }^{n *}$ are homogeneous of degree $r+1$, and so on respectively in $\xi^{*}$.

Thus $g_{i * h}$ and $g_{i^{*} h^{*}}$ must be homogeneous of degree $q-1$ and $q-2$ respectively in $\xi^{*}$ if $g_{i h}$ are homogeneous of degree $q$. But, since we have (4.3), $g_{i^{*} h}$ must be homogeneous of degree 0 . Thus we have

Proposition 4.3. The components of the almost metric tensor field stated in Proposition 4.2 are homogeneous in $\xi^{*}$ of degree

$$
\begin{array}{lll}
g_{i h}:+1, & g_{i^{*} h}: 0, & g_{i^{*} h *}:-1, \\
g^{i n}:-1, & g^{i * h}: 0, & g^{i * h^{*}}:+1 .
\end{array}
$$

\section{$\S 5$. Fundamental function and fundamental hypersurfaces.}

Definition. Let $c$ be an arbitrary positive constant. A hypersurface determined by the equation

$$
g_{B A} p^{B} p^{A}=c
$$


namely,

$$
g^{i \hbar} \xi^{i^{*}} \xi^{h^{*}}=c
$$

is called a fundamental hypersurface.

Definition. The function

$$
F=g^{i n} \xi^{i^{*}} \xi^{h^{*}}
$$

is called the fundamental function.

The fundamental function is homogeneous of degree 1 in $\xi^{*}$.

Assumption A. We assume that the even-dimensional contact manifold $M$ satisfies the condition that the vector field $p^{A}$ is orthogonal to the fundamental hypersurfaces.

Let $u^{A}$ be an arbitrary vector field which is tangent to the fundamental hypersurfaces. Then $\boldsymbol{u}^{\boldsymbol{A}}$ satisfies

$$
\left(\partial_{j} g^{i h}\right) \xi^{i^{*}} \xi^{h^{*}} u^{j}+\left\{\left(\partial_{j^{*}} g^{i h}\right) \xi^{i^{*}} \xi^{h^{*}}+2 g^{j h} \xi^{h^{*}}\right\} u^{j^{*}}=0 .
$$

On the other hand, as the vector fields $p^{A}$ and $u^{A}$ are mutually orthogonal, we have

$$
g_{j h * \xi^{*}} u^{j}+g_{j^{*} h} \xi^{h^{*}} u^{j^{*}}=0 .
$$

Hence we get, by eliminating $u^{A}$,

$$
\begin{aligned}
& \left(\partial_{j} g^{i n}\right) \xi^{i^{*}} \xi^{h^{*}}=\lambda g_{j h *} \xi^{h^{*}}, \\
& \left(\partial_{j *} g^{i h}\right) \xi^{i^{*}} \xi^{h^{*}}+2 g^{j h} \xi^{h^{*}}=\lambda g^{j h} \xi^{h^{*}},
\end{aligned}
$$

where we have used (4.2).

From the last equation we get $\lambda=1$ as we have $\xi^{j^{*}} \partial_{\jmath^{*}} g^{i h}=-g^{i h}$.

Thus we have proved

PROPOSITION 5.1. A necessary and sufficient condition in order that the natural vector field $p^{A}$ be parallel to the normal vector field of the fundamental hypersurfaces is that the following equations be satisfied,

$$
\begin{gathered}
\left(\partial_{j *} g^{i h}\right) \xi^{i^{*}} \xi^{h^{*}}=-g^{j h} \xi^{h^{*}}, \\
\partial_{j} g^{i n} \xi^{i^{*}} \xi^{h^{*}}=-g^{j^{*} h} \xi^{h^{*}} .
\end{gathered}
$$

This condition is also equivalent to

$$
\partial_{j} F=g_{j A} p^{A}, \quad \partial_{j *} F=g_{j * A} p^{A}
$$

or

$$
\partial_{A} F=g_{B A} p^{B} .
$$

$\S 6$. Orthogonality of distributions $\mathscr{Q}$ and $Q$.

In the sequel we consider an even-dimensional contact manifold $M$ which 
admits a contact almost product structure $H=P-Q$ and assume A. We put further assumptions one by one.

Assumption B. We assume that the even-dimensional contact manifold $M$ with the contact almost product structure $H=P-Q$ introduced in $\S 2$ admits a pair of symmetric tensor fields $t_{B A}$ and $t^{B A}$ such that

$$
t_{B C} t^{C A}=\delta_{B}^{A}
$$

and

$$
f_{B C} t^{C A}=p_{B}{ }^{A}-q_{B}{ }^{A} \text {. }
$$

If we write the condition (6.2) in non-holonomic components, we have

$$
F_{B C} T^{C A}=\left[\begin{array}{rr}
E & 0 \\
0 & -E
\end{array}\right],
$$

where $F_{B A}$ satisfies (3.5). Hence we have

$$
\begin{aligned}
T^{i h} & =T^{i^{*} h^{*}}=0, \\
T^{i^{*} h} & =T^{i h^{*}}=-\delta_{i h}
\end{aligned}
$$

and

$$
\begin{aligned}
T_{i h} & =T_{i * h^{*}}=0, \\
T_{i * h} & =T_{i h^{*}}=-\delta_{i h} .
\end{aligned}
$$

Assumption C. The tensor fields $t_{B A}, t^{B A}$ and $g^{B A}$ satisfy

$$
t^{B A}=g^{B D} g^{A C} t_{D C} \text {. }
$$

In non-holonomic components we can write (6.5) in the form

$$
T^{B A}=G^{B D} G^{A C} T_{D C}
$$

where $T^{B A}$ and $T_{B A}$ are given by (6.3) and (6.4) respectively. Hence we have

$$
\begin{aligned}
& -G^{i k} G^{h k^{*}}-G^{i k^{*}} G^{h k}=0, \\
& -G^{i * k} G^{h k^{*}}-G^{i^{*} k^{*}} G^{h k}=-\delta_{i h} .
\end{aligned}
$$

On the other hand, as the non-holonomic components $F_{B A}$ and the holonomic components $f_{B A}$ satisfy the same equations (2.6) and (3.5), the non-holonomic components $G^{B A}$ satisfy

$$
\begin{aligned}
& G^{i^{*} k} G^{k^{*} h}-G^{i^{*} k^{*}} G^{k h}=-\delta_{i h}, \\
& G^{i k} G^{k^{*} h}-G^{i k^{*}} G^{k h}=0 .
\end{aligned}
$$

Hence we get

$$
G^{i k} G^{k^{*} h}=0, \quad G^{i^{*} k} G^{k^{*} h}=0
$$

and consequently 


$$
G^{i^{*} h}=0 .
$$

This implies that the distributions $\mathscr{P}$ and $Q$ are mutually orthogonal.

Conversely, if we have (6.6), we get $(6.5)$.

Thus we have proved

Proposition 6.1. Let $M$ be an even-dimensional contact manifold with a contact almost product structure and an almost metric tensor field $g_{B A}$ such that $f_{B C} g^{O A}$ is an almost complex structure. Let us assume that there exists in $M$ globally a pair of symmetric tensor fields $t_{B A}$ and $t^{B A}$ such that $f_{B C} t^{C A}$ is the tensor field of the contact almost product structure $p_{B}{ }^{A}-q_{B}{ }^{A}$ and that $t_{B C} t^{C A}=\delta_{B}^{A}$. Then $t_{B A}$ and $t^{B A}$ satisfy $t_{B A}=g_{B D} g_{A C} t^{D C}$ if and only if the distributions $\mathscr{P}$ and $Q$ are orthogonal.

It will be immediately seen that

$$
G_{i h}=G^{i^{*} h^{*}}, \quad G_{i * h^{*}}=G^{i h} .
$$

\section{§7. Affine connection.}

In the sequel we assume $\mathrm{A}, \mathrm{B}$ and $\mathrm{C}$. Hence $M$ satisfies all conditions stated in Proposition 6. 1.

Let us assume that $M$ admits an affine connection $\gamma$. We denote its components by $\gamma_{C^{A}}{ }_{B}$ or $\Gamma_{C}{ }_{B}{ }_{B}, \Gamma_{C}{ }_{B}{ }_{B}$ being non-holonomic components.

Assumption D. The affine connection $\gamma$ is such that the distributions $\mathscr{Q}$ and $Q$ are parallel with respect to $\gamma$.

This means that, if $l^{A}$ is a vector field satisfying $q_{B}{ }^{A} l^{B}=0$, then $l^{A}$ satisfies

$$
q_{B}{ }^{A}\left(\partial_{C} l^{B}+\gamma c^{B}{ }_{K} l^{K}\right)=0
$$

and also that, if $m^{A}$ is a vector field satisfying $p_{B}{ }^{A} m^{B}=0$, then $m^{A}$ satisfies

$$
p_{B}{ }^{A}\left(\partial_{C} m^{B}+\gamma C^{B}{ }_{K} m^{K}\right)=0 .
$$

As we have $p_{B}{ }^{C} q_{C}{ }^{A}=0, q_{B}{ }^{C} p_{C}{ }^{A}=0$ and $p_{B}{ }^{A}+q_{B}{ }^{A}=\delta_{B}^{A}$, the above condition is equivalent to

$$
\nabla_{C} p_{B}{ }^{A}=0, \quad \nabla_{C} q_{B}{ }^{A}=0,
$$

where $\nabla_{C}$ is the symbol of covariant differentiation with respect to $\gamma$.

The relation between the holonomic components $\gamma \sigma^{A}{ }_{B}$ and the non-holonomic components $\Gamma_{C}{ }^{A}{ }_{B}$ being

$$
\gamma_{C^{A}}{ }_{B}=\left(\partial_{C} Z_{B}{ }^{H}\right) Y_{H}{ }^{A}+\Gamma_{L}{ }^{H}{ }_{K} Y_{H}{ }^{A} Z_{C}{ }^{L} Z_{B}{ }^{K},
$$

$\nabla_{C} p_{B}{ }^{A}=0$ can be written in the form

$$
\nabla_{C} P_{B}{ }^{A} \equiv D_{C} P_{B}{ }^{A}+\Gamma_{C}{ }_{K}{ }_{K} P_{B}{ }^{K}-\Gamma_{C}{ }_{B}{ }_{B} P^{A}=0 .
$$

$P_{B}{ }^{A}$ is given by (3.4) and we have $\Gamma_{C}{ }^{h^{*}}{ }_{2}=0$. From $\nabla_{C} q_{B}{ }^{A}=0$ we get similarly $\Gamma_{e^{n}{ }^{n}}=0$. 
Thus we have

Proposition 7.1. Let $M$ be an even-dimensional contact manifold with a contact almost product structure $p_{B}{ }^{A}-q_{B}{ }^{A}$ and let there be given an affine connection in $M$. A necessary and sufficient condition for the distributions $\mathscr{P}$ and $Q$ to be parallel is that the non-holonomic components of the affine connection satisfy

$$
\Gamma_{C^{h}}{ }^{h^{*}}=0, \quad \Gamma_{C^{*}}{ }^{*}=0 .
$$

We have assumed that this condition is satisfied. We put further assumptions.

Let us denote the torsion tensor of the connection by $s_{C B}{ }^{A}$ or $S_{C B}{ }^{A}$.

Assumption E. The components of the torsion tensor in each of the distributions $\mathscr{P}$ and $Q$ vanish, namely,

$$
s_{L K}{ }^{H} p_{C}{ }^{L} p_{B}{ }^{K} p_{H}{ }^{A}=0, \quad s_{L K}{ }^{H} q_{C}{ }^{L} q_{B}{ }^{K} q_{H}{ }^{A}=0 .
$$

This assumption bears quite natural meaning.

As we have

$$
S_{L K}^{H}=\frac{1}{2}\left[-\left(D_{L} Y_{K}^{B}-D_{K} Y_{L}^{B}\right) Z_{B}{ }^{H}+\Gamma_{L}{ }^{H}-\Gamma_{K}{ }^{H} L\right]
$$

from (7.2), we can write (7.4) in the form

$$
\begin{aligned}
& \left(D_{k} Y_{j}^{A}-D_{\jmath} Y_{k}{ }^{A}\right) Z_{A}{ }^{h}-\Gamma_{k^{k}}{ }_{j}+\Gamma_{j}{ }^{h} k_{k}=0, \\
& \left(D_{k^{*}} Y_{j^{*}}{ }^{A}-D_{j^{*}} Y_{k^{*}}{ }^{A}\right) Z_{A}{ }^{h^{*}}-\Gamma_{k^{*}}{ }^{h^{*}}{ }^{*}+\Gamma_{j^{*}}{ }^{h^{*}}{ }_{k^{*}}=0,
\end{aligned}
$$

or

$$
\begin{aligned}
& \Gamma_{k^{\prime}}{ }_{j}-\Gamma_{j}{ }^{h}{ }_{k}=\left(D_{k} \Gamma_{j l}-D_{j} \Gamma_{k l}\right) \Pi^{i h}, \\
& \Gamma_{k^{*}}{ }^{h^{*}{ }^{*}}-\Gamma_{j^{*}}{ }^{h^{*}}{ }_{k^{*}}=-\Pi^{j l} D_{k^{*}} \Gamma_{l h}+\Pi^{k l} D_{j^{*}} \Gamma_{l h} \text {. }
\end{aligned}
$$

Assumption F. The skew-symmetric tensor field $f_{B A}$ is covariantly constant with respect to the connection $\gamma$.

We immediately find that this is equivalent to

$$
\Gamma_{C}{ }_{i}+\Gamma_{C^{i *}}{ }^{h^{*}}=0
$$

for we have already $\Gamma_{C^{2}}{ }^{h^{*}}=\Gamma_{C^{*}}{ }_{i *}=0$.

Assumption G. The affine connection $\gamma$ is conformal with respect to the tensor field $g_{B A}$ in each of the distributions $\mathscr{P}$ and $Q$.

This means that the following equations hold.

$$
\begin{aligned}
& \nabla_{C}\left(g_{L K} p_{B}{ }^{L} p_{A}{ }^{K}\right)=a_{C} g_{L K} p_{B}{ }^{L} p_{A}{ }^{K}, \\
& \nabla_{C}\left(g_{L K} q_{B}{ }^{L} q_{A}{ }^{K}\right)=b_{C} g_{L K} q_{B}{ }^{L} q_{A}{ }^{K} .
\end{aligned}
$$

As $p_{B}{ }^{A}$ and $q_{B}{ }^{A}$ are covariantly constant and $G_{i h^{*}}=0,(7.9)$ is equivalent to 


$$
\begin{aligned}
& D_{C} G_{i h}-\Gamma_{C}{ }_{i} G_{k h}-\Gamma_{C}{ }_{h} G_{i k}=A_{C} G_{i h},
\end{aligned}
$$

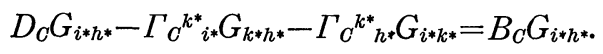

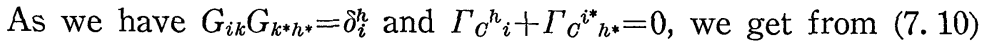

$$
B_{C}=-A_{C} \text {. }
$$

We write here the non-holonomic components of the torsion tensor for later use.

$$
S_{j i}^{h}=0
$$

$$
S_{j i}^{h^{*}}=-\frac{1}{2}\left(D_{j} \Gamma_{i h}-D_{i} \Gamma_{j h}\right)
$$

$$
S_{j * i}^{h}=-\frac{1}{2}\left[\left(D_{j *} \Gamma_{i k}\right) \Pi^{k h}+D_{i} \Pi^{j h}+\Pi^{j l}\left(\partial_{i} \Gamma_{l k}\right) \Pi^{k h}\right]+\frac{1}{2} \Gamma_{j * \imath}^{h}
$$

Here, (7.12) and (7.17) are equations (7.4) themselves. Others are obtained from (7. 5) by using (3.2), (3.3), (7.3) and (7.8).

\section{§ 8. Almost Finsler manifold.}

Let $P^{A}$ be the non-holonomic components of the natural vector field.

Assumption H. $P^{A}$ satisfy

$$
\begin{gathered}
P^{h}=0, \\
S_{C B}{ }^{A} P^{C}=0 .
\end{gathered}
$$

(8. 1) is equivalent to

$$
\Pi \Pi^{j i} \xi^{j^{*}}=0
$$

and means that the natural vector field lies in the distribution $Q$. As (8.2) is equivalent to

$$
s_{C B}^{A} p^{C}=0,
$$

its geometrical implication will be immediate.

Substituting (7.14), (7.15), (7.16) and $P^{j^{*}}=\xi^{j^{*}}$ into (8.2) we get

$$
\Gamma_{j *}{ }^{h} \xi^{j^{*}}=0 \text {, }
$$

$$
\Gamma_{i h}=\Gamma_{i^{j}} \xi^{j^{*}}
$$

for we have 


$$
\begin{gathered}
\xi^{j^{*}} D_{j^{*}}=\xi^{j^{*}} \partial_{j^{*},} \\
\left(D_{i} \Pi^{j h}\right) \xi^{j^{*}}=-\Pi^{j h} D_{i \xi^{j^{*}}}=-\Pi^{j h} \Gamma_{\imath \jmath}, \\
\xi^{j^{*}} \partial_{j^{*}} \Pi^{i h}=-\Pi^{i h}, \\
\xi^{j^{*}} \partial_{j^{*}} \Gamma_{i h}=\Gamma_{i h} .
\end{gathered}
$$

The relation $S_{j * i i^{*}} \xi^{\xi^{*}=0}$ is identically satisfied.

Now the non-holonomic components $F_{B}{ }^{A}$ of the almost complex structure are

$$
F_{i}{ }^{h}=0, \quad F_{i}^{h^{*}}=-G_{i h}, \quad F_{i *^{h}}=G^{i h}, \quad F_{i *^{n^{*}}}=0
$$

because of (6.6). Let $U^{A}$ be a contravariant vector and $\tilde{U}^{A}$ the vector $\tilde{U}^{A}=F_{B}{ }^{A} U^{B}$, then

$$
\tilde{U}^{h}=G^{i n} U^{i^{*}}, \quad \tilde{U}^{h^{*}}=-G_{i h} U^{i} .
$$

The vectors $U^{A}$ and $\tilde{U}^{A}$ span a holomorphic plane. If $U^{A}$ is a vector of $\mathscr{Q}(Q)$, then $\tilde{U}^{A}$ is a vector of $Q(\mathscr{Q})$.

Assumption I. The vector $s_{C B}{ }^{A}\left(v^{C} \tilde{u}^{B}-u^{C} \tilde{v}^{B}\right)$ is a vector of $Q$ when $u^{A}$ and $v^{A}$ are vectors of $\mathscr{L}$.

This is equivalent to

$$
S_{j k^{*}}{ }^{n} F_{i}{ }^{*}-S_{i k^{*}}{ }^{n} F_{j} k^{*}=0
$$

or to

$$
S_{j * k}^{h} G^{k \imath}-S_{i * k}^{h} G^{k \jmath}=0
$$

by virtue of (8.5).

Assumption $I$ is also equivalent to

Assumption I'. The vector $s_{C B}{ }^{A}\left(v^{C} u^{B}-\tilde{v}^{C} \tilde{u}^{B}\right)$ is a vector of $Q$ when one of the vectors $u, v$ is a vector of $\mathscr{Q}$ and the other is a vector of $Q$.

Assumption J. The scalar field $F$ satisfies

$$
\nabla_{C}\left(F^{-1} g_{L K} p_{B}^{L} p_{A}^{K}\right)=0 .
$$

This is equivalent to

$$
a_{C}=\partial_{C} \log F
$$

or to

$$
A_{C}=F^{-1} D_{C} F
$$

by virtue of (7.9).

Let us write

$$
p_{A}=g_{B A} p^{B}, \quad P_{A}=G_{B A} P^{B} .
$$

Then, since we have $P^{h}=0$ and $P^{h^{*}}=\xi^{h^{*}}$, we get $P_{A}=G_{k^{*} A} \xi^{k^{*}}$, and hence

$$
P_{\imath}=0, \quad P_{i^{*}}=G^{i k} \xi^{k^{*}} \text {. }
$$


Using (5.4) we also obtain

$$
D_{A} F=P_{A}, \quad D_{i *} F=\partial_{i *} F^{1)}
$$

Thus we get

$$
A_{\jmath}=0, \quad A_{j^{*}}=\left(G^{l k} \xi^{l^{*}} \xi^{k^{*}}\right)^{-1} G^{j i} \xi^{i^{*}} .
$$

We collect here all that we have assumed.

$M$ is an even-dimensional contact manifold with contact almost product structure and almost metric structure $g_{B A}$. These are such that $M$ becomes an almost complex manifold and that the distributions $\mathscr{P}$ and $Q$ induced by the almost product structure are orthogonal with respect to the almost metric. Such structures are homogeneous in the sense that the contact frames $\Gamma_{j i}$ and $\Pi^{i n}$ are homogeneous functions of degree 1 and -1 respectively in $\xi^{1^{*}}, \cdots, \xi^{n^{*}}$ and that the components $g_{B A}$ of the almost metric structure are such that $g_{i h}, g_{i^{*} h}, g_{i^{*} h^{*}}$ are homogeneous of degree $1,0,-1$ respectively in $\xi^{1^{*}}, \cdots, \xi^{n^{*}}$. The natural vector field $p^{A}$ is parallel to the normal vector field of the fundamental hypersurfaces which are defined by $F=$ constant where $F=g_{B A} p^{B} p^{A}$. The affine connection $\gamma$ defined over $M$ is such that the distributions $\mathscr{Q}$ and $Q$ are parallel with respect to $\gamma$ and the skew symmetric tensor field of the homogeneous contact structure is covariantly constant with respect to $\gamma \cdot \gamma$ is symmetric and conformal in each of the distributions $\mathscr{P}$ and $Q$. The natural vector field belongs to the distribution $Q$. Let $S$ denote the torsion tensor of the connection $\gamma$ so that $S(u, v)=-S(v, u)$ for any vectors $u$ and $v$. Then $S(p, v)$ vanishes identically when $p$ is the natural vector field. At any point $\mathrm{P}$ of $M$ let $u$ be a vector of $\mathscr{Q}$ and $v$ a vector of $Q$. Let $\tilde{u}$ and $\tilde{v}$ be the vectors obtained from $u$ and $v$ by the operation of the almost complex structure of $M$ respectively. Then $S(u, v)-S(\tilde{u}, \tilde{v})$ is a vector of $Q$. The almost metric structure induced in $\mathscr{Q}$ becomes a metric structure when multiplied by $F^{-1}$.

A manifold which satisfies all conditions stated above will be called an almost Finsler manifold.

\section{§9. The connection in an almost Finsler manifold.}

In an almost Finsler manifold $\Gamma_{j i}, \Pi^{i h}$ and $G_{j i}$ are related in a complicated way. If we put

$$
\begin{aligned}
\sum_{j i} h & =\frac{1}{2}\left(D_{j} \Gamma_{i k}-D_{i} \Gamma_{j k}\right) \Pi^{k h}, \\
\sum_{j^{* i *}}{ }^{*} & =\frac{1}{2}\left(-\Pi^{i k} D_{j^{*}} \Gamma_{k h}+\Pi^{j k} D_{i^{*}} \Gamma_{k h}\right),
\end{aligned}
$$

then we can write some of the coefficients $\Gamma_{C}{ }_{B}{ }_{B}$ in the following form

$$
\Gamma_{j}{ }_{\imath}{ }_{\imath}=\frac{1}{2} G^{h l}\left(D_{j} G_{i l}+D_{i} G_{j l}-D_{l} G_{j i}\right)+\sum_{j i}{ }^{h}-\sum_{j}{ }_{i}-\sum_{i}{ }^{h}{ }_{j},
$$

1) Notice that this is obtained from the assumption that the distributions $\mathscr{Q}$ and $Q$ are mutually orthogonal. 
(9. 4)

$$
\Gamma_{j^{*}{ }^{h^{*}}{ }^{*}}=\frac{1}{2} G^{h^{*} l^{*}}\left\{\left(D_{j^{*}}+A_{j^{*}}\right) G_{i^{*} l^{*}}+\left(D_{i^{*}}+A_{i^{*}}\right) G_{j^{* *} l^{*}}-\left(D_{l^{*}}+A_{l^{*}}\right) G_{j v^{*}}\right\}
$$

$$
+\sum_{j * i *} h^{*}-\sum_{J^{*}}{ }^{h^{*}}{ }^{*}-\sum_{i^{*}}{ }^{h^{*}}{ }^{*}
$$

where

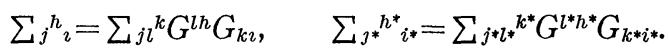

Hence, if we consider that $\Gamma_{j i}, \Pi^{i n}$ and $G_{j i}$ are given, we can determine all coefficients $\Gamma_{C}{ }_{B}{ }_{B}$, using (7.3) and (7.8). Substituting the coefficients thus obtained into (8.3), (8.4) and (8.7) we get conditions which must be satisfied by $\Gamma_{j i}, \Pi^{i h}$ and $G_{j i}$.

Let us first consider the condition (8. 3).

From (9.4) we get

$$
\begin{aligned}
& \Gamma_{j^{*}}{ }^{h^{*}}{ }_{{ }^{*} \xi^{*}} j^{j^{*}}=\frac{1}{2} G^{h^{*} l^{*}}\left\{\xi^{j^{*}} D_{j^{*}} G_{i^{*} l^{*}}+\xi^{j^{*}}\left(D_{i^{*}} G_{j^{*} l^{*}}-D_{l^{*}} G_{j^{*} i^{*}}\right)\right\}
\end{aligned}
$$

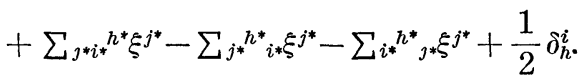

But we have

$$
\begin{aligned}
\xi^{j^{*}} D_{i^{*}} G_{j^{*} l^{*}} & =D_{i^{*}}\left(\xi^{j^{*}} G_{j^{*} l^{*}}\right)-G_{j^{*} l^{*}} D_{i^{*}} \xi^{j^{*}} \\
& =D_{i^{*}}\left(\xi^{j^{*}} G^{j l}\right)-G^{j l}\left(\delta_{j}^{i}-\Pi \Pi^{i t} \Gamma_{t j}\right) \\
& =D_{i^{*}} \partial_{l^{*}} F-G^{i l}+\Pi^{i t} \Gamma_{t j} G^{j l} \\
& =\left(\partial_{i^{*}}-\Pi^{i s} \partial_{s}-\Pi^{i t} \Gamma_{t s} \partial_{s^{*}} \partial_{l^{*}} F-G^{i l}+\Pi^{i t} \Gamma_{t j} G^{j l}\right. \\
& =\partial_{i^{*}} \partial_{l^{*}} F-\Pi^{i s} \partial_{l^{*}} \partial_{s} F-\Pi^{i t} \Gamma_{t s} \partial_{s^{*}} \partial_{l^{*}} F-G_{i l}+\Pi^{i t} \Gamma_{t j} G^{j l} .
\end{aligned}
$$

As we have $P_{i}=0$, that is, $D_{i} F=0$, we can substitute

$$
\partial_{i} F=-\Gamma_{i t} \partial_{t^{*}} F
$$

into the above formula and get

$$
\begin{aligned}
\xi^{j^{*}} D_{i^{*}} G_{j^{*} l^{*}} & =\partial_{i^{*}} \partial_{l^{*}} F+\Pi^{i s} \partial_{l^{*}}\left(\Gamma_{s t} \partial_{t^{*}} F\right)-\Pi^{i t} \Gamma_{t s} \partial_{l^{*}} \partial_{s^{*}} F-G^{i l}+\Pi^{i t} \Gamma_{t j} G^{j l} \\
& =\partial_{i^{*}} \partial_{l^{*}} F+\Pi^{i s} \partial_{l^{*}} \Gamma_{s t} \partial_{t^{*}} F-G^{i l}+\Pi^{i t} \Gamma_{t j} G^{j l},
\end{aligned}
$$

hence

$$
\begin{aligned}
& \xi^{j^{*}}\left(D_{i^{*}} G_{l^{*} l^{*}}-D_{l^{*}} G_{j^{*} i^{*}}\right) \\
= & \left(\Pi^{i s} \partial_{l^{*}} \Gamma_{s j}-\Pi^{l s} \partial_{i^{*}} \Gamma_{s j}\right) \partial_{j^{*}} F+\Pi^{i t} \Gamma_{t s} G^{s l}-\Pi^{l t} \Gamma_{t s} G^{s \imath} .
\end{aligned}
$$

On the other hand we have 


$$
\begin{aligned}
& \left(\Pi^{i s} D_{l^{*}} \Gamma_{s j}-\Pi^{l s} D_{i^{*}} \Gamma_{s j}\right) \partial_{j^{*}} F \\
= & \left\{\Pi^{i s}\left(\partial_{l^{*}}-\Pi^{l t} \partial_{t}-\Pi^{l r} \Gamma_{r t} \partial_{t^{*}}\right) \Gamma_{s j}-\Pi^{l s}\left(\partial_{i^{*}}-\Pi^{i t} \partial_{t}-\Pi^{i r} \Gamma_{r t} \partial_{t^{*}}\right) \Gamma_{s j}\right\} \partial_{j^{*}} F \\
= & \left(\Pi^{i s} \partial_{l^{*}} \Gamma_{s j}-\Pi^{l s} \partial_{l^{*}} \Gamma_{s j}\right) \partial_{j^{*}} F-\left(\Pi^{i s} \Pi^{l t} \partial_{t} \Gamma_{s j}-\Pi^{l s} \Pi^{i t} \partial_{t} \Gamma_{s j}\right) \partial_{j^{*}} F \\
& -\left(\Pi^{i s} \Pi^{l r} \Gamma_{r t} \partial_{t^{*}} \Gamma_{s j}-\Pi^{l s} \Pi^{i r} \Gamma_{r t} \partial_{t^{*}} \Gamma_{s j}\right) \partial_{j^{*}} F \\
= & \left(\Pi^{i s} \partial_{l^{*}} \Gamma_{s j}-\Pi^{l s} \partial_{i^{*}} \Gamma_{s j}\right) \partial_{j^{*}} F \\
& -\left(\Pi^{i s} \Pi^{l t}-\Pi^{l s} \Pi^{i t}\right)\left\{\partial_{t}\left(\Gamma_{s j} \partial_{j^{*}} F\right)-\Gamma_{s j} \partial_{j^{*}} \partial_{t} F+\Gamma_{t r} \partial_{r^{*}} \Gamma_{s j} \partial_{j^{*}} F\right\} \\
= & \left(\Pi^{i s} \partial_{l^{*}} \Gamma_{s j}-\Pi^{l s} \partial_{i^{*}} \Gamma_{s j}\right) \partial_{j^{*} F} F \\
& -\left(\Pi^{i s} \Pi^{l t}-\Pi^{l s} \Pi^{i t}\right)\left\{-\partial_{t} \partial_{s} F+\Gamma_{s j} \partial_{j^{*}}\left(\Gamma_{t r} \partial_{r^{*}} F\right)+\Gamma_{t j} \partial_{j^{*}} \Gamma_{s r} \partial_{r^{*}} F\right\} \\
= & \left(\Pi^{i s} \hat{\partial}_{l^{*}} \Gamma_{s j}-\Pi^{l s} \partial_{i^{*}} \Gamma_{s j}\right) \partial_{j^{*}} F .
\end{aligned}
$$

Thus we get

$$
\xi^{j^{*}}\left(D_{i^{*}} G_{j^{*} l^{*}}-D_{l^{*}} G_{j^{* *}}\right)=\Pi^{i t} \Gamma_{t s} G^{s l}-\Pi^{l t} \Gamma_{t s} G^{s 2}+2 \sum_{i^{*} l^{*}}{ }^{j^{*}} P_{j^{*}}
$$

Using this identity and

$$
\begin{aligned}
& \sum_{j * i *}{ }^{h^{*} \xi^{j^{*}}}=-\frac{1}{2} \Pi^{i k} \Gamma_{k h}, \\
& \sum_{J^{*}{ }^{k^{*}}{ }_{i *} \xi^{j^{*}}}=-\frac{1}{2} \Pi^{t k} \Gamma_{k s} G_{t h} G^{s \imath},
\end{aligned}
$$

we get

$$
\Gamma_{j *}{ }^{h *}{ }_{i *} \xi^{j^{*}}=0 .
$$

Thus (8. 3) is automatically satisfied and the only essential conditions are (8.4) and (8.7).

Let us write (8.7) in another form.

We get from (8.7)

$$
\begin{aligned}
& {\left[-\left(D_{j^{*}} \Gamma_{k l}\right) \Pi^{l h}-D_{k} \Pi^{j h}-\Pi^{\jmath m} \Pi^{l h} \partial_{k} \Gamma_{m l}\right] G_{k^{*} i^{*}}} \\
& -\left[-\left(D_{i^{*}} \Gamma_{k l}\right) \Pi^{l h}-D_{k} \Pi^{i n}-\Pi^{i m} \Pi^{l h} \partial_{k} \Gamma_{m l}\right] G_{k^{*} j *}=\Gamma_{j^{*}}{ }^{k^{*}}{ }^{*} G_{k^{*} i *}-\Gamma_{\imath^{*}}{ }^{*}{ }_{h^{*}} G_{k^{*} j^{*}},
\end{aligned}
$$

and, as we have

$$
\begin{aligned}
& D_{j^{*}} G_{i^{*} h^{*}}-D_{i^{*}} G_{j^{*} l^{*}}
\end{aligned}
$$

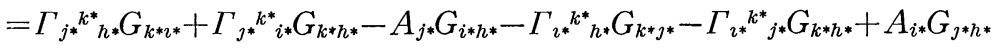

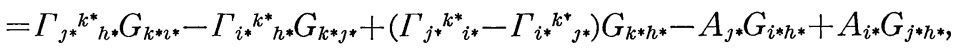

we obtain

$$
\begin{aligned}
& D_{\jmath^{*}} G_{i^{*} h^{*}}-D_{i^{*}} G_{j^{*} h^{*}} \\
= & {\left[-\left(D_{\jmath^{*}} \Gamma_{k l}\right) \Pi^{l h}-D_{k} \Pi^{j h}-\Pi^{\jmath m} \Pi^{i h} \partial_{k} \Gamma_{m l}\right] G_{k^{*} i^{*}} } \\
& -\left[-\left(D_{i^{*}} \Gamma_{k l}\right) \Pi^{i h}-D_{k} \Pi^{i h}-\Pi^{i m} \Pi^{l h} \partial_{k} \Gamma_{m l}\right] G_{k^{*} j *} \\
& +\left(-\Pi^{i l} D_{\jmath^{*}} \Gamma_{l k}+\Pi^{j l} D_{i^{*}} \Gamma_{l k}\right) G_{k^{*} h^{*}}-A_{\jmath^{*}} G_{i^{*} h^{*}}+A_{\imath^{*}} G_{\jmath^{*} h^{*}}
\end{aligned}
$$


We write here the non-holonomic components of the covariant derivative of the natural vector field. These are

$$
\begin{aligned}
& \nabla_{j} P^{h}=D_{j} P^{h}+\Gamma_{j}{ }^{h}{ }^{*} P^{i^{*}}=0, \\
& \nabla_{j^{*}} P^{h}=D_{j *} P^{h}+\Gamma_{j^{*}}{ }^{h}{ }^{*} P^{i^{*}}=0, \\
& \nabla_{j} P^{h^{*}}=D_{j} P^{h^{*}+}+\Gamma_{j}{ }^{h^{*}}{ }^{*} P^{i^{*}}=\Gamma_{j h}-\Gamma_{j}{ }^{2} \xi^{i^{*}}=0
\end{aligned}
$$

and

$$
\nabla_{j^{*}} P^{h^{*}}=D_{j^{*}} P^{h^{*}}+\Gamma_{j^{*}}^{h^{*}}{ }^{*} P^{i^{*}}=\delta_{h}^{j}-\Pi^{j i} \Gamma_{i h}+\Gamma_{j^{*}}{ }^{h^{*}}{ }_{i *} \xi^{i^{*}} .
$$

But we have

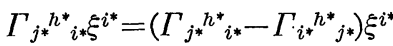

$$
\begin{aligned}
& =2 \sum_{j * i *}{ }^{* *} \xi^{i^{*}}=\Pi^{j k} \Gamma_{k h} .
\end{aligned}
$$

Hence $\nabla_{j *} P^{h^{*}}=\delta_{h}^{j}$ and we get

$$
\nabla_{B} P^{A}=Q_{B}{ }^{A}
$$

\section{$\S 10$. The case in which the distribution $Q$ is completely integrable.}

Let us examine the case in which the distribution $Q$ is completely integrable.

$Q$ is represented by $\omega^{2}=0$, and, since the system $\omega^{2}=0$ is equivalent to the system $p_{B}{ }^{A} d \xi^{B}=0$, this case is characterized by

$$
q_{C}{ }^{L} q_{B}{ }^{K}\left(\partial_{L} q_{K}^{A}-\partial_{K} q_{L}^{A}\right)=0,
$$

which is equivalent to

$$
Q_{C}^{T} Y_{T}^{L} Q_{B}^{S} Y_{S}^{K}\left(\partial_{L} q_{K}^{A}-\partial_{K} q_{L}^{A}\right)=0
$$

because of $q_{B}{ }^{A}=Z_{B}{ }^{T} Q_{T}{ }^{S} Y_{S}{ }^{A}$. As we have $Q_{B}{ }^{A}=\delta_{B}^{k^{*}} \delta_{k^{*}}^{A}$, we get

$$
Y_{j *}^{L} Y_{i^{*}}{ }^{K}\left(\partial_{L} q_{K}^{A}-\partial_{K} q_{L}^{A}\right)=0 \text {, }
$$

and hence

$$
Y_{j *}^{L} D_{i *} q_{L}^{A}-Y_{i^{*}}^{L} D_{j *} q_{L}^{A}=0 .
$$

After some straightforward calculation we find that the latter is equivalent to

$$
D_{j *} \Pi^{i n}-D_{i^{*}} \Pi^{j h}+\left(\Pi^{i l} D_{j^{*}} \Gamma_{l k}-\Pi^{j l} D_{i^{*}} \Gamma_{l k}\right) \Pi^{k h}=0 .
$$

Let us assume that $\operatorname{det}\left(\delta_{i}^{h}-\Pi^{h l} \Gamma_{l i}\right)$ does not vanish. Then we can show that there exists a homogeneous contact transformation $\left(\xi^{\imath}, \xi^{i^{*}}\right) \rightarrow\left(\zeta^{i}, \zeta^{i^{*}}\right)$ such that the manifolds $\zeta^{h}=c^{h}$ are the integral manifolds of $Q$ in the local sense.

When $Q$ is completely integrable, the system of partial differential equations

$$
q_{B}{ }^{A} \partial_{A} \zeta=0
$$

is completely integrable. Since (10.3) is equivalent to

$$
-\Pi^{h k} \partial_{k} \zeta+\left(\delta_{k}^{h}-\Pi^{h l} \Gamma_{l k}\right) \partial_{k^{*}} \zeta=0,
$$


where $\operatorname{det}\left(\delta_{i}^{h}-\Pi^{h l} \Gamma_{l i}\right) \neq 0$ by assumption, $\partial_{i *} \zeta$ are determined when $\partial_{i} \zeta$ are given. Hence there exists a set of $n$ solutions $\zeta^{h}$ of (10.3) such that

$$
\frac{\partial\left(\zeta^{1}, \cdots, \zeta^{n}\right)}{\partial\left(\xi^{1}, \cdots, \xi^{n}\right)} \neq 0 .
$$

Besides, since we have $\Pi^{i n} \xi^{h^{*}}=0$, we find that these solutions satisfy

$$
\xi^{i^{*}} \partial_{i *} \zeta^{h}=0
$$

which proves that $\zeta^{n}$ are homogeneous functions of degree 0 in $\xi^{*}$.

Let us determine $\zeta^{h^{*}}$ from the equations

$$
\zeta^{h^{*}} \partial_{i} \zeta^{h}=\xi^{i^{*}}
$$

Transvecting $\zeta^{h^{*}}$ to the equations

$$
-\Pi^{i k} \partial_{k} \zeta^{h}+\left(\delta_{k}^{i}-\Pi^{i l} \Gamma_{l k}\right) \partial_{k^{*}} \zeta^{h}=0
$$

we get

$$
\left(\delta_{k}^{2}-\Pi^{i l} \Gamma_{l k}\right)\left(\zeta^{h^{*}} \partial_{k^{*}} \zeta^{h}\right)=0
$$

hence

$$
\zeta^{h^{*}} \partial_{k^{*}} \zeta^{h}=0
$$

by virtue of $\operatorname{det}\left(\delta_{k}^{2}--\Pi^{i l} \Gamma_{l k}\right) \neq 0$.

This proves that, if we take $\zeta^{h}$ satisfying (10.5) and (10.7) and determine $\zeta^{h^{*}}$ by $(10.6)$, then $\left(\xi^{i}, \xi^{i *}\right) \rightarrow\left(\zeta^{i}, \zeta^{i^{*}}\right)$ is a homogeneous contact transformation.

We can also prove that $\zeta^{h}$ satisfy

$$
\operatorname{det}\left(\partial_{i} \zeta^{h}+\Gamma_{i k} \partial_{k^{*}} \zeta^{h}\right) \neq 0 .
$$

As we are dealing with local properties, we can restrict our consideration to that at each point $\mathrm{P}$ of $M$.

Let the rank of $\Pi^{i n}$ be $n-m$ at $\mathrm{P}$ and let us use indices as follows: $\alpha, \beta=1, \cdots, m ; \kappa, \lambda=m+1, \cdots, n$. We choose $\zeta^{\alpha}$ in such a way that

$$
\Pi^{i k} \partial_{k} \zeta^{\alpha}=0
$$

and $\zeta^{x}$ that (10.5) holds.

Suppose $\tau_{h}$ satisfy

$$
\tau_{h}\left(\partial_{i} \zeta^{h}+\Gamma_{i k} \partial_{k^{*}} \zeta^{h}\right)=0
$$

Transvecting such $\tau_{h}$ to (10.7) we obtain

$$
\tau_{h} \partial_{i * \zeta^{h}}=0
$$

and hence $\Pi^{i k_{\tau}} \tau_{h} \partial_{k} \zeta^{h}=0$ by (10.7). By virtue of (10.9) we get $\Pi^{i k_{k}} \partial_{k} \partial_{k} \zeta^{x}=0$. But, since $\zeta^{h}$ satisfy (10.5), $\tau_{x}$ must vanish and we get

$$
\tau_{\alpha} \partial_{i *} \zeta^{\alpha}=0
$$

from (10.11). Thus (10.10) takes the form $\tau_{\alpha} \partial_{i} \zeta^{\alpha}=0$ from which we get $\tau_{\alpha}=0$ because of (10.5). Hence $\tau_{h}$ must vanish. This proves (10.8). 
Thus $\left(\zeta^{i}, \zeta^{*}\right)$ is a canonical contact coordinate system $[8,13,14,22]$.

The distribution $Q$ is expressed by $d \zeta^{h}=0$ in this coordinate system $\left(\zeta^{i}, \zeta^{i^{*}}\right)$. Hence the second contact trame must vanish. ing

As $\operatorname{det}\left(\delta_{i}^{h}-\Pi^{h l} \Gamma_{l i}\right) \neq 0$ is equivalent to $\tilde{\omega} \wedge d \xi^{1^{*}} \wedge \cdots \wedge d \xi^{n *} \neq 0$, we obtain the follow-

Proposition 10.1. Let $M$ be an even-dimensional contact manifold admitting a contact almost product structure such that $\tilde{\omega} \wedge d \xi^{1^{*}} \wedge \cdots \wedge d \xi^{n^{*}} \neq 0$. Let the distribution $Q$ be completely integrable. Then there exists a set of canonical contact coordinate systems covering $M$ such that the second contact frame vanishes identically.

We shall use in the sequel only such contact coordinate systems and denote them by $\left(\xi, \xi^{*}\right)$.

\section{$\S 11$. Finsler metric.}

Let $M$ be a space in which we can use canonical contact coordinate systems such that $\Pi^{i n}=0$. Any transformation $\left(\xi^{2}, \xi^{i^{*}}\right) \rightarrow\left(\xi^{i^{\prime}}, \xi^{i^{*}}\right)$ between such coordinate systems takes the form

$$
\xi^{h^{\prime}}=\xi^{h^{\prime}}\left(\xi^{1}, \cdots, \xi^{n}\right), \quad \xi^{n^{* \prime}}=\xi^{h^{* \prime}}\left(\xi^{1}, \cdots, \xi^{n} ; \xi^{1^{*}}, \cdots, \xi^{n^{*}}\right),
$$

for $\xi^{h^{\prime}}$ must satisfy $\partial_{i^{*}} \xi^{h^{\prime}}=0$.

Let us define $\stackrel{\circ}{G}_{i n}$ and $\stackrel{\circ}{G}^{i n}$ by

$$
\stackrel{\circ}{G}_{i h}=\left(G^{l k} \xi^{l^{*}} \xi^{k^{*}}\right)^{-1} G_{i h}=F^{-1} G_{i h},
$$

$$
\stackrel{\circ}{G}^{i n}=\left(G^{l k} \xi^{* *} \xi^{k^{*}}\right) G^{i n}=F G^{i n} .
$$

These are homogeneous functions of degree 0 in $\xi^{*}$. We then define $x^{n}$ by

$$
\stackrel{*}{x}^{n}=\stackrel{\circ}{G}^{i n} \xi^{i^{*}}, \quad \xi^{i^{*}}=\stackrel{\circ}{G}_{i h}{ }^{*} x^{n} .
$$

We can consider $F=G^{l k} \xi^{k^{*}} \xi^{k^{*}}$ as a function of $\xi^{1}, \cdots, \xi^{n} ; x^{*}, \cdots, *^{n}$. In general, when we regard a function $\varphi\left(\xi, \xi^{*}\right)$ of the independent variables $\xi^{1}, \cdots, \xi^{n} ; \xi^{1^{*}}, \cdots, \xi^{n^{*}}$ as a function of the new independent variables $\xi^{1}, \cdots, \xi^{n} ; *^{* 1}, \cdots, *^{*}$, we shall use the letters $x^{\imath}$ for $\xi^{\imath}$. Thus we can write

$$
F\left(\xi, \xi^{*}\right)=F(x, \stackrel{*}{x})=\sqrt{\dot{G}_{i h} x^{i} x^{*}}=G^{i n} \xi^{i^{*}} \xi^{h^{*}} .
$$

We continue to use the symbols $\partial_{i}$ and $\partial_{i^{*}}$ for

$$
\partial_{i}=\frac{\partial}{\partial \xi^{i}}, \quad \partial_{i *}=\frac{\partial}{\partial \xi^{i^{*}}}
$$

with the understanding that the independent variables are $\xi^{1}, \cdots, \xi^{n} ; \xi^{1 *}, \cdots, \xi^{n *}$. When we use $x^{1}, \cdots, x^{n} ; \stackrel{*}{x^{1}}, \cdots, *^{*}$ as independent variables, we do not use abbreviated notations but write 


$$
\frac{\partial}{\partial x^{2}}, \quad \frac{\partial}{\partial x^{*}} .
$$

As the function $F$ plays an important role, it might be better to use the symbol $F_{i^{*}}$ for $P_{i^{*}}$, and hence

$$
\stackrel{*}{x}^{h}=F F_{h^{*}}
$$

We also have

$$
\stackrel{*}{x}^{n} \xi^{h^{*}}=F^{2}=\stackrel{\circ}{G}_{i h} *^{*} \stackrel{*}{x}^{n} .
$$

(11. 4) is equivalent to

$$
\partial_{h *} F^{2}=2 x^{* h} .
$$

On the other hand, from (11.5) we get

$$
\partial_{h^{*}} F^{2}=\stackrel{*}{x}^{h}+\xi^{i^{*}} \frac{\partial x^{*} i}{\partial \xi^{h^{*}}} .
$$

Thus we get

$$
\stackrel{*}{x}^{h}=\xi^{i^{*}} \frac{\partial *^{\imath}}{\partial \xi^{h^{*}}} .
$$

As we can write (11.4) in the form

$$
\stackrel{*}{x}^{h}=F \frac{\partial F}{\partial x^{*} i} \frac{\partial *^{*} i}{\partial \xi^{h^{*}}},
$$

we obtain

$$
\xi^{h^{*}}=F \frac{\partial F}{\partial x^{*} h},
$$

where we assume

$$
\operatorname{det}\left(\frac{\partial x^{* h}}{\partial \xi^{i^{*}}}\right) \neq 0
$$

This assumption is equivalent to

$$
\operatorname{det}\left(\partial_{\jmath *} \partial_{i *}\left(\frac{1}{2} F^{2}\right)\right) \neq 0
$$

by virtue of (11. 4).

We can prove that $\partial_{k^{*}}\left(F G^{j i}\right)$ is symmetric with respect to the indices $i, j, k$.

As we have $\Pi^{i h}=0$, we get from (9.5)

$$
\partial_{j *} G^{i n}-\partial_{i *} G^{j h}=-A_{j *} G^{i n}+A_{i *} G^{j h},
$$

and hence

(11. 9)

$$
\partial_{k^{*}}\left(F G^{j i}\right)-\partial_{j^{*}}\left(F G^{k i}\right)=0
$$

because of $A_{j *}=F^{-1} F_{j *}$ 
Differentiating $\stackrel{*}{x}^{h}=F G^{i n} \xi^{i^{*}}$ and taking account of (11.9) we get

$$
\begin{aligned}
\frac{\partial x^{* h}}{\partial \xi^{j^{*}}} & =\frac{\partial\left(F G^{i n}\right)}{\partial \xi^{j^{*}}} \xi^{i^{*}}+F G^{i h} \\
& =\frac{\partial\left(F G^{j h}\right)}{\partial \xi^{i^{*}}} \xi^{i^{*}}+F G^{j h} \\
& =F G^{j h}
\end{aligned}
$$

since $F G^{j h}$ are homogeneous of degree 0 in $\xi^{*}$. Thus we obtain

$$
\begin{aligned}
& \frac{\partial \mathscr{*}^{h}}{\partial \xi^{i^{*}}}=\stackrel{\circ}{G}^{i n}, \\
& \frac{\partial \xi^{h^{*}}}{\partial \mathscr{x}^{*}}=\stackrel{\circ}{G}_{i h .} .
\end{aligned}
$$

Differentiating (11.7) with respect to ${ }^{*}{ }^{i}$ and using (11.11) we get

$$
\frac{1}{2} \frac{\partial^{2} F^{2}}{\partial x^{*} \partial x^{*}}=\stackrel{\circ}{G}_{i h}
$$

which shows that $\stackrel{\circ}{G}_{i h}(x, \stackrel{*}{x})$ determine a Finsler metric for the element of support $(x, \stackrel{*}{x})$.

\section{$\S 12$. Finsler space.}

We have shown that, in the manifold $M$ considered in $\S 11$, the metric $\stackrel{\circ}{G}_{i n}$ is a Finsler metric for the element of support $(x, *)$ in the local sense. Now let us assume that the set of the integral manifolds of the distribution $Q$ determines a fibering of the manifold $M$ such that $M$ becomes a bundle space of a fibre bundle $\mathfrak{M}=\{M, X, \pi, F\}$ with the following properties. The base space $X$ is an $n$-dimensional manifold whose points are expressed locally by $\left(\xi^{1}, \cdots, \xi^{n}\right)$, the projection $\pi$ is given by $\pi:\left(\xi^{1}, \cdots, \xi^{n} ; \xi^{1^{*}}, \cdots, \xi^{n *}\right) \rightarrow\left(\xi^{1}, \cdots, \xi^{n}\right)$ and the fibre $F$ is $R^{n}-\{0\}$. But this does not mean that $F$ is a Euclidean space with a point 0 excluded. We only assume that $\xi^{1^{*}}, \cdots, \xi^{n *}$ can take any set of real numbers except $\xi^{1^{*}}=\cdots=\xi^{n^{*}}=0$.

Then there exists a Finsler space $\mathfrak{M}^{*}=\left\{M^{*}, X, \pi^{*}, F^{*}, G\right\}$ which is related to $\mathfrak{M}$ in the following way.

Let $x$ be a point of $X$ and let $F_{x}$ and $F_{x}^{*}$ be the fibres over $x$ respectively of $\mathfrak{M}$ and $\mathfrak{M}^{*}$. A point of $F_{x}$ is denoted by $\left(\xi^{1}, \cdots, \xi^{n} ; \xi^{1^{*}}, \cdots, \xi^{n *}\right)$ and a point of $F_{x}^{*}$ by $\left(\xi^{1}, \cdots, \xi^{n} ; \stackrel{*}{x}^{1}, \cdots, *^{*}\right)$ or $\left(x^{1}, \cdots, x^{n} ; \stackrel{*}{x}^{1}, \cdots, *^{* n}\right)$ where $x^{\imath}=\xi^{\imath}$. $\pi^{*}$ is the projection $\pi^{*}:\left(x^{1}, \cdots, x^{n} ; x^{1}, \cdots, *^{n}\right) \rightarrow\left(x^{1}, \cdots, x^{n}\right)$ and $\stackrel{\circ}{G}$ means the Finsler metric. There exists a diffeomorphic mapping $\varphi$ of $\mathfrak{M}$ onto $\mathfrak{M}^{*}$ such that $\varphi:\left(\xi^{1}, \cdots, \xi^{n} ; \xi^{1^{*}}, \cdots, \xi^{n *}\right)$ $\rightarrow\left(\xi^{1}, \cdots, \xi^{n} ; *^{*}, \cdots, *^{n}{ }^{n}\right)$ is given by $(11.2)$.

Let us consider in $\mathfrak{M}^{*}$ the Finsler connection due to E. Cartan and study its relation to the connection $\gamma$ in $M$.

The coefficients of the Cartan connection will be denoted by $\Pi_{j}{ }_{\imath} \imath_{\imath}$ and $C_{j}{ }^{h}$ 乞 so that if we use the symbol 


$$
\left\{\begin{array}{c}
h \\
j i
\end{array}\right\}=\frac{1}{2} \stackrel{\circ}{G}^{h l}\left(\frac{\partial \stackrel{\circ}{G l}_{j l}}{\partial x^{2}}+\frac{\partial \stackrel{\circ}{G}_{i l}}{\partial x^{j}}-\frac{\partial \stackrel{\circ}{G i}_{j i}}{\partial x^{l}}\right) \text {, }
$$

we have

$$
\begin{aligned}
& \Pi_{j}{ }^{h}=\left\{\begin{array}{c}
h \\
j i
\end{array}\right\}-C_{l}{ }^{h}{ }_{j} \frac{\partial G^{l}}{\partial x^{*}}+C_{l i j} \frac{\partial G^{l}}{\partial x^{*}} F G^{h m}, \\
& C_{j}{ }^{h} i=F G^{h k} C_{j i k} \\
& =\frac{1}{2} F G^{h k} \frac{\partial\left(F^{-1} G_{j i}\right)}{\partial x^{*}} \\
& =\frac{1}{2} F G^{h k} \partial_{l^{*}}\left(F^{-1} G_{j i}\right) \frac{\partial \xi^{l^{*}}}{\partial x^{* k}} \\
& =\frac{1}{2} \partial_{h^{*}}\left(F^{-1} G_{j i}\right) \text {, }
\end{aligned}
$$

where

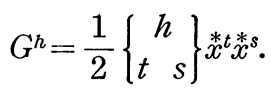

If $\mathfrak{D}^{*}$ denotes the covariant differential in the sense of Cartan, we have

$$
\mathfrak{D}^{*} U^{h}=d U^{h}+C_{j}{ }^{h} i\left(x, *^{*}\right) U^{i} d x^{*}+\Pi_{j}^{h} i\left(x, *^{*}\right) U^{i} d x^{\jmath} .
$$

Now let us consider the tangent bundle $T(\mathscr{P})$ over $M$. For each point $\mathrm{P} \in M$, $T_{\mathrm{P}}(\mathscr{P})$ is the vector space spanned by the tangent vectors of $\mathscr{P}$ at $\mathrm{P}$. Thus, if $l \in T_{\mathrm{P}}(\mathscr{P})$, we have

$$
l^{h}=L^{h}, \quad l^{h^{*}}=\Gamma_{h k} L^{k},
$$

namely, $L^{h^{*}}=0$, where $L^{A}$ are the non-holonomic components. As $\mathscr{Q}$ is parallel with respect to the affine connection $\gamma$ of $M$, we can construct from the differentials $d L^{A}$ and $d \xi^{A}$ the classical absolute differential $\mathscr{D} L^{A}$ by

$$
\mathscr{D} L^{A}=d L^{A}+\Gamma_{C}{ }^{A} \omega^{C} L^{B},
$$

where

$$
\mathscr{D} L^{h}=d L^{h}+\Gamma{ }_{c^{h}}{ }_{i} \omega^{C} L^{2}, \quad \mathfrak{D} L^{h^{*}}=0
$$

by virtue of $L^{h^{*}}=0$ and (7.3). We further obtain

$$
\mathfrak{D} L^{h}=d L^{h}+\Gamma_{j}{ }^{h}{ }_{i} d \xi^{j} L^{\imath}+\Gamma_{j *}{ }^{h}{ }_{i}\left(d \xi^{j^{*}}-\Gamma_{j k} d \xi^{k}\right) L^{\imath}
$$

or

$$
\mathscr{D} L^{h}=d L^{h}+\left(\Gamma_{j}{ }_{i}-\Gamma_{j k} \Gamma_{k^{*}}{ }_{i}{ }_{i}\right) d \xi^{j} L^{\imath}+\Gamma_{j *}{ }^{h}{ }_{i} d \xi^{j *} L^{\imath} .
$$

On the other hand we can extend the mapping $\varphi$ to the vector fields in the following sense. 
If $l$ is a tangent vector of $\mathscr{Q}$ at $\mathrm{P} \in M$, then $\varphi l$ is the vector $U$ at $\varphi \mathrm{P} \in M^{*}$ whose components $U^{h}$ are given by $U^{h}=L^{h}$ where $L^{A}$ are the non-holonomic components of $l$.

Such a mapping induces a connection in $\mathfrak{M}^{*}$ which can be expressed in the form of absolute differential

$$
\begin{aligned}
\mathfrak{D} U^{h} & =d U^{h}+\Gamma_{j}{ }_{i}{ }_{i} d \xi^{j} U^{i}+\Gamma_{j *}{ }^{h}\left(d \xi^{j^{*}}-\Gamma_{j k} d \xi^{k}\right) U^{i} \\
& =d U^{h}+\left(\Gamma_{j}{ }_{i}-\Gamma_{j k} \Gamma_{k^{*}}{ }^{h}\right) d \xi^{j} U^{i}+\Gamma_{j^{*}}{ }_{i} d \xi^{j^{*}} U^{i} .
\end{aligned}
$$

If we get

$$
\mathfrak{D} U^{h}=\mathfrak{D} * U^{h}
$$

by substituting (11.2) into (12.5), then we can say that we could obtain a Finsler manifold with the Cartan connection from an almost Finsler manifold whose distribution $Q$ is completely integrable.

The remaining part of the paper is devoted to the proof of (12.7).

As we have

$$
\begin{aligned}
& d x^{*}=\frac{\partial *^{*} h}{\partial \xi^{i^{*}}} d \xi^{i^{*}}+\frac{\partial x^{*} h}{\partial \xi^{\imath}} d \xi^{\imath}, \\
& d x^{h}=d \xi^{h}
\end{aligned}
$$

where

$$
\frac{\partial x^{* h}}{\partial \xi^{i^{*}}}=F G^{i h}, \quad \frac{\partial x^{*} h}{\partial \xi^{i}}=\frac{\partial\left(F G^{h l} \xi^{l^{*}}\right)}{\partial \xi^{i}}
$$

(12. 7) is equivalent to

$$
\begin{aligned}
& F C_{k}{ }_{i} G^{k \jmath}=\Gamma_{\jmath^{*}}{ }_{\imath}, \\
& \Pi_{j}{ }^{h}{ }_{i}+C_{l}{ }_{\imath}{ }_{\imath} \frac{\partial\left(F G^{l k} \xi^{k^{*}}\right)}{\partial \xi^{j}}=\Gamma_{j}{ }^{h}{ }_{i}-\Gamma_{j k} \Gamma_{k^{*}{ }^{h}{ }^{h} .}
\end{aligned}
$$

\section{$\S 13$. Proof of (12.7).}

Proof of (12.8) is quite simple.

As we have $\Pi^{i h}=0$, we get $\sum_{\jmath^{*} i{ }^{h *}}{ }^{*}=0$ in (9.4). Hence we have

$$
\begin{aligned}
\Gamma_{j^{*}}^{h^{*}} i^{*}= & \frac{1}{2} G_{h l}\left(\partial_{j^{*}} G^{i l}+\partial_{i^{*}} G^{j l}-\partial_{l^{*}} G^{j i}\right) \\
& +\frac{1}{2} F^{-1} G_{h l}\left(F_{j^{*}} G^{i l}+F_{i^{*}} G^{j l}-F_{l^{*}} G^{j i}\right) \\
= & \frac{1}{2} F^{-1} G_{h l}\left\{\partial_{j^{*}}\left(F G^{i l}\right)+\partial_{i^{*}}\left(F G^{j l}\right)-\partial_{l^{*}}\left(F G^{j i}\right)\right\}
\end{aligned}
$$


As $\partial_{j^{*}}\left(F G^{i l}\right)$ is symmetric in $i, j, l$, we get

$$
\begin{aligned}
\Gamma_{\jmath^{*}{ }^{h^{*}}} & =\frac{1}{2} F^{-1} G_{h l} \partial_{i^{*}}\left(F G^{j l}\right), \\
\Gamma_{\jmath^{*}{ }^{h}} & =-\frac{1}{2} F^{-1} G_{i l} \partial_{h^{*}}\left(F G^{j l}\right) .
\end{aligned}
$$

On the other hand we get from (12.3)

$$
\begin{aligned}
F C_{k}{ }_{i}{ }_{i} G^{k j} & =\frac{1}{2} \partial_{h^{*}}\left(F^{-1} G_{k i}\right) F G^{k j} \\
& =-\frac{1}{2} F^{-1} G_{i l} \partial_{h^{*}}\left(F G^{j l}\right),
\end{aligned}
$$

which proves (12.8).

(12.9) is equivalent to

$$
\Gamma_{j}{ }^{n} i-\Gamma_{j k} \Gamma_{k^{*}}{ }^{n}
$$

$$
=\left\{\begin{array}{c}
h \\
j
\end{array}\right\}-C_{l^{h}}{ }^{h} \frac{\partial G^{l}}{\partial x^{*}}+C_{l i \jmath} \frac{\partial G^{l}}{\partial x^{* m}} F G^{h m}+C_{k}^{h} i \frac{\partial x^{* k}}{\partial \xi^{\jmath}} .
$$

Let us define $\left[\begin{array}{c}h \\ j\end{array}\right]$ by

$$
\left[\begin{array}{c}
h \\
j
\end{array}\right]=\frac{1}{2} G^{h l}\left(\partial_{j} G_{i l}+\partial_{i} G_{j l}-\partial_{l} G_{j i}\right)
$$

and prove

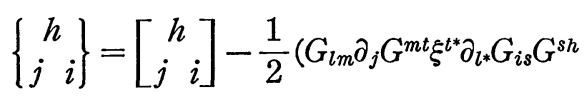

$$
\begin{aligned}
& +G_{l m} \partial_{i} G^{m t} \xi^{t^{*}} \partial_{l^{*}} G_{j s} G^{s h} \\
& \left.-G_{l m} \partial_{s} G^{m t} \xi^{t^{*}} \partial_{l^{*}} G_{j i} G^{s h}\right),
\end{aligned}
$$

where $\partial_{j}=\frac{\partial}{\partial \xi^{\jmath}}$.

Since we have

$$
\frac{\partial \xi^{k^{*}}}{\partial x^{*} l} \frac{\partial *^{*} l}{\partial \xi^{j}}+\frac{\partial \xi^{k^{*}}}{\partial x^{l}} \frac{\partial x^{l}}{\partial \xi^{j}}=0
$$

and $x^{i}=\xi^{i}$, we get from (11.2)

$$
\begin{aligned}
\frac{\partial \xi^{k^{*}}}{\partial x^{j}} & =-F^{-1} G_{k l} \frac{\partial x^{*}}{\partial \xi^{i}} \\
& =-F^{-1} \partial_{j} F \xi^{k^{*}}-G_{k l} \partial_{j} G^{l t} \xi^{*},
\end{aligned}
$$


hence

$$
\frac{\partial}{\partial x^{j}}=\partial_{j}-\left(F^{-1} \partial_{j} F \xi^{l^{*}}+G_{l m} \partial_{j} G^{m t} \xi^{l^{*}}\right) \partial_{l^{*}}
$$

and

$$
\begin{aligned}
\frac{\partial F}{\partial x^{j}} & =\partial_{j} F-\left(F^{-1} \partial_{j} F \xi^{l^{*}}+G_{l m} \partial_{j} G^{m t} \xi^{\xi^{*}}\right) G^{l s} \xi^{s^{*}} \\
& =-\partial_{j} F .
\end{aligned}
$$

Substituting these identities into (12.1) and calculating we get (13.5). In order to prove (13.3) we must first calculate $G^{h}$.

From (13.5) we get

$$
\begin{aligned}
G^{h}= & \left.\frac{1}{2}\left[\begin{array}{c}
h \\
j
\end{array}\right]\right]^{*^{j} x^{*}} \\
& +\frac{1}{2} F^{* j} \partial_{j} G^{m t} G_{m l} \xi^{*} \xi^{*} \partial_{l^{*}} G^{s h} \\
& +\frac{1}{4} F^{2} G^{h s} \partial_{s} F
\end{aligned}
$$

Substituting (13.4) into this formula and using the properties of $G_{j i}$ given by (11.9), we get

$$
\begin{aligned}
G^{h}= & -\frac{1}{2} F^{2}\left(\partial_{r} G^{h s}\right) G^{r t} \xi^{t^{*}} \xi^{s^{*}} \\
& -\frac{1}{2} F G^{t s} \xi^{t^{*}} \partial_{s} F F_{h^{*}}+\frac{1}{2} F^{2} G^{h s} \partial_{s} F
\end{aligned}
$$

and

$$
\begin{aligned}
\partial_{k^{*}} G^{h}= & -\frac{1}{2} F^{2} \partial_{t} G^{h s} G^{t k} \xi^{s^{*}}-\frac{1}{2} F^{2} \partial_{s} G^{h k} F_{s^{*}} \\
& +F F_{k^{*}} G^{h s} \partial_{s} F+\frac{1}{2} F^{2} \partial_{k^{*}} G^{h s} \partial_{s} F \\
& +\frac{1}{2} F^{2} G^{h s} \partial_{s} F_{k^{*}}-\frac{1}{2} F G^{s k} \partial_{s} F F_{h^{*}} \\
& -\frac{1}{2} F F_{s^{*}} \partial_{s} F G^{h k} .
\end{aligned}
$$

In this calculation and also in the following calculation we often use the fact that $\partial_{k^{*}}\left(F G^{j i}\right)$ is symmetric in $i, j, k$ and also the identities

$$
\begin{aligned}
\partial_{j *} G^{i n} & =F^{-1}\left(F_{i *} G^{j h}-F_{j *} G^{i n}\right)+\partial_{i^{*}} G^{j h}, \\
\partial_{j^{*}} G^{i t} \xi^{*} & =-F^{-1} F_{j^{*}} F_{i^{*} .}
\end{aligned}
$$


From (12.3) and (13.8) we obtain after straightforward calculation

$$
\begin{aligned}
& -C_{l}^{h}, \frac{\partial G^{l}}{\partial x^{*} i}+C_{l i j} \frac{\partial G^{l}}{\partial x^{* m}} F G^{h m} \\
& =\frac{1}{2 F^{2}} F_{h^{*}}\left[-\frac{F}{2} \partial_{i} G^{l s} \xi^{s^{*}} G_{l j}+\frac{F}{2} \partial_{r} G_{j i} F_{r^{*}}+\xi^{i^{*}} \partial_{j} F\right. \\
& \left.+\frac{F}{2} G_{l j} G_{k i} \partial_{k^{*}} G^{l s} \partial_{s} F+\frac{F}{2} G_{i k} \partial_{j} G^{k t \xi^{t^{*}}}\right] \\
& -\frac{1}{2 F} \partial_{h^{*}} G_{l j}\left[-\frac{F}{2} \partial_{i} G^{l s} \xi^{\xi^{*}}-\frac{F}{2} G_{k i} \partial_{r} G^{l k} F_{r^{*}}+\xi^{i^{*}} G^{l s} \partial_{s} F\right. \\
& \left.+\frac{F}{2} G_{k i} \partial_{k^{*}} G^{l s} \partial_{s} F+\frac{F}{2} G^{l s} G_{i k} \partial_{s} G^{k t} \xi^{t^{*}}\right] \\
& +\frac{1}{4 F} G_{j i}\left(\partial_{r} F G^{r h}-\frac{1}{F} F_{h^{*}} F_{s^{*}} \partial_{s} F\right) \\
& +\frac{1}{2 F} G_{l p} \partial_{p^{*}} G_{j i}\left[-\frac{F}{2} \partial_{r} G^{l s} \xi^{s^{*}} G^{r h}-\frac{F}{2} \partial_{r} G^{l h} F_{r^{*}}\right. \\
& \left.+F_{h^{*}} G^{l s} \partial_{s} F+\frac{F}{2} \partial_{h^{*}} G^{l s} \partial_{s} F+\frac{F}{2} G^{l s} \partial_{s} G^{h t} \xi^{t^{*}}\right] .
\end{aligned}
$$

Another term in the right hand side of (13.3) is obtained in the form

$$
\begin{aligned}
C_{k}{ }_{i} i \frac{\partial x^{*}}{\partial \xi^{j}} & =\frac{1}{2} \frac{\partial\left(F^{-1} G_{k \imath}\right)}{\partial \xi^{h^{*}}} \partial_{j}\left(F G^{k l} \xi^{l^{*}}\right) \\
& =-\frac{1}{2 F}\left(F_{h^{*}} G_{k i}-F \partial_{h^{*}} G_{k i}\right) \partial_{j} G^{k l} \xi^{l^{*}} .
\end{aligned}
$$

From these formulas we find that the right hand side of (13. 3) is equal to

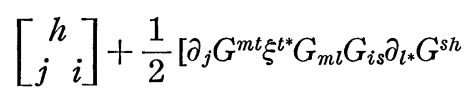

$$
\begin{aligned}
& +\partial_{i} G^{m t} \xi^{* *} G_{m l} G_{j s} \partial_{l^{*}} G^{s h} \\
& \left.+G^{h s} \partial_{s} G^{m t} \xi^{t^{*}} G_{m l} \partial_{l^{*}} G_{j i}\right] \\
& +\frac{F_{h^{*}}}{2 F^{2}}\left[-\frac{F}{2} \partial_{i} G^{l s \xi^{*}} G_{l j}+\frac{F}{2} \partial_{r} G_{j i} F_{r^{*}}\right. \\
& +\xi^{i^{*}} \partial_{j} F+\frac{F}{2} G_{l j} G_{k i} \partial_{k^{*}} G^{l s} \partial_{s} F \\
& \left.+\frac{F}{2} G_{i k} \partial_{j} G^{k t} \xi^{t^{*}}\right]
\end{aligned}
$$


HOMOGENEOUS CONTACT MANIFOLDS AND ALMOST FINSLER MANIFOLDS

$$
\begin{gathered}
-\frac{1}{2 F} \partial_{h^{*}} G_{l \jmath}\left[-\frac{F}{2} \partial_{i} G^{l s \xi^{*}}-\frac{F}{2} G_{k i} \partial_{r} G^{l k} F_{r^{*}}\right. \\
+\xi^{i^{*}} G^{l s} \partial_{s} F+\frac{F}{2} G_{k i} \partial_{k^{*}} G^{l s} \partial_{s} F \\
\left.+\frac{F}{2} G^{l s} G_{i k} \partial_{s} G^{k t} \xi^{l^{*}}\right] \\
+\frac{1}{4 F} G_{j i}\left(\partial_{r} F G^{r h}-\frac{1}{F} F_{h^{*}} F_{s^{*}} \partial_{s} F\right) \\
+\frac{1}{2 F} G_{l p} \partial_{p^{*}} G_{j i}\left[-\frac{F}{2} \partial_{r} G^{l s} \xi^{s^{*}} G^{r h}-\frac{F}{2} \partial_{r} G^{l h} F_{r^{*}}\right. \\
+F_{h^{*}} G^{l s} \partial_{s} F+\frac{F}{2} \partial_{h^{*}} G^{l s} \partial_{s} F \\
\left.+\frac{F}{2} G^{l s} \partial_{s} G^{h t} \xi^{t^{*}}\right] \\
-\frac{1}{2 F}\left(F_{h^{*}} G_{k i}-F \partial_{h^{*}} G_{k i}\right) \partial_{j} G^{k l \xi \xi^{*} .}
\end{gathered}
$$

Let us calculate the left hand side of (13.3).

As we have $\Pi^{j i}=0, \Gamma_{j}{ }^{h}$ is symmetric with respect to $i, j$ as we can see from (7. 6). As we have $A_{\imath}=0$ also, we get from (9.3)

hence

$$
\Gamma_{j}{ }^{h}{ }_{\imath}=\frac{1}{2} G^{h l}\left(D_{j} G_{i l}+D_{i} G_{j l}-D_{l} G_{j i}\right)
$$

$$
\Gamma_{j \imath}^{h}=\left[\begin{array}{c}
h \\
j
\end{array}\right]+\frac{1}{2} G^{h l}\left(\Gamma_{\jmath m} \partial_{m^{*}} G_{i l}+\Gamma_{\imath m} \partial_{m^{*}} G_{j l}-\Gamma_{l m} \partial_{m^{*}} G_{j i}\right) .
$$

As we have $\Gamma_{i l} F_{l^{*}}=-\partial_{i} F$, we get

$$
\begin{aligned}
& I_{j i}=\Gamma_{j}{ }^{h}{ }_{i} \xi^{h^{*}}
\end{aligned}
$$

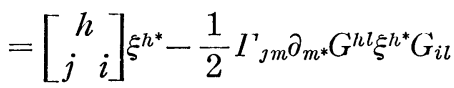

$$
\begin{aligned}
& -\frac{1}{2} \Gamma_{\imath m} \partial_{m^{*}} G^{h l} \xi^{h^{*}} G_{j l}+\frac{1}{2} \partial_{m} F \partial_{m^{*}} G_{j i} \\
& =\left[\begin{array}{c}
h \\
j
\end{array}\right]\left[\xi^{h^{*}}-\frac{1}{2 F}\left(\partial_{j} F \xi^{i^{*}}+\partial_{i} F \xi^{j^{*}}\right)+\frac{1}{2} \partial_{m} F \partial_{m^{*}} G_{j i}\right.
\end{aligned}
$$

hence

$$
\Gamma_{j i}=\frac{1}{2}\left(\partial_{j} G_{i t}+\partial_{i} G_{j t}-\partial_{t} G_{j i}\right) F_{t^{*}}
$$

(13. 10)

$$
-\frac{1}{2 F}\left(\partial_{j} F \xi^{i^{*}}+\partial_{i} F \xi^{j^{*}}\right)+\frac{1}{2} \partial_{t} F \partial_{t^{*}} G_{j i}
$$


Thus we get the complete expression of $\Gamma_{j}{ }^{h}$,

$$
\begin{aligned}
& \Gamma_{j}{ }^{h}=\left[\begin{array}{c}
h \\
j
\end{array}\right] \\
& -\frac{1}{2} \partial_{m^{*}} G^{h l} G_{i l}\left[\frac{1}{2}\left(\partial_{j} G_{m t}+\partial_{m} G_{j t}-\partial_{t} G_{j m}\right) F_{t^{*}}\right. \\
& \left.-\frac{1}{2 F}\left(\partial_{j} F \xi^{m^{*}}+\partial_{m} F \xi^{j^{*}}\right)+\frac{1}{2} \partial_{t} F \partial_{t^{*}} G_{j m}\right] \\
& -\frac{1}{2} \partial_{m^{*}} G^{h l} G_{j l}\left[\frac{1}{2}\left(\partial_{i} G_{m t}+\partial_{m} G_{i t}-\partial_{t} G_{i m}\right) F_{t^{*}}\right. \\
& \left.-\frac{1}{2 F}\left(\partial_{i} F \xi^{m^{*}}+\partial_{m} F \xi^{i^{*}}\right)+\frac{1}{2} \partial_{t} F \partial_{t^{*}} G_{i m}\right] \\
& -\frac{1}{2} G^{h l} \partial_{m^{*}} G_{j i}\left[\frac{1}{2}\left(\partial_{m} G_{l t}+\partial_{l} G_{m t}-\partial_{t} G_{m l}\right) F_{t^{*}}\right. \\
& \left.-\frac{1}{2 F}\left(\partial_{l} F \xi^{m^{*}}+\partial_{m} F \xi^{l^{*}}\right)+\frac{1}{2} \partial_{t} F \partial_{t^{*}} G_{m l}\right] .
\end{aligned}
$$

We get from (13.2)

$$
-\Gamma_{k^{*} \imath}{ }^{h}=\frac{1}{2 F} G_{i l} \partial_{k^{*}}\left(F G^{h l}\right)
$$

and hence

$$
\begin{aligned}
-\Gamma_{j k} \Gamma_{k^{*}}{ }_{i}=\frac{1}{2 F} G_{i l} \partial_{m^{*}}\left(F G^{h l}\right) & {\left[\frac{1}{2}\left(\partial_{j} G_{m t}+\partial_{m} G_{j t}-\partial_{t} G_{\jmath m}\right) F_{t^{*}}\right.} \\
& \left.-\frac{1}{2 F}\left(\partial_{j} F \xi^{m^{*}}+\partial_{m} F \xi^{j^{*}}\right)+\frac{1}{2} \partial_{t} F \partial_{t *} G_{\jmath m}\right] .
\end{aligned}
$$

Using these expressions we can prove (13.3) by straightforward calculation.

Thus we have

Theorem 13.1. Let $M$ be an almost Finsler manifold such that the distribution $Q$ is completely integrable. Let the integral manifolds of $Q$ be such that there exists a fibre bundle $\mathfrak{M}=\{M, X, \pi, F\}$. Then there exists a Finsler space $\mathfrak{M}^{*}=\left\{M^{*}, X, \pi^{*}, F^{*}, \stackrel{\circ}{G}\right\}$, where the mapping $\varphi: \mathfrak{M} \rightarrow \mathfrak{M}^{*}$ is given by (11.2) and the connection induced from $\gamma$ by $\varphi$ is the Cartan connection.

\section{REFERENCES}

[1] Boothby, W. M., And H. C. Wang, On contact manifolds. Ann. Math. 68 (1958), 721-734.

[2] Cartan, E., Leçons sur les invariants intégraux. Parıs, Hermann, 1922.

[3] Cartan, E., Les espaces métriques fondés sur la notion d'aire. Act. Sci. Industr. 
72. Paris, Hermann, 1933.

[4] Cartan, E., Les espaces de Finsler. Act. Sc1. Industr. 79. Paris, Hermann, 1934.

[5] CARTAN, E., Les systèmes différentiels extérieurs et leurs applications géométr1ques. Act. Sc1. Industr. 994. Parıs, Hermann, 1945.

[6] Doyle, T. C., Tensor decomposition with applications to the contact and complex groups. Ann. Math. 42 (1941), 698-721.

[7] Eisenhart, L. P., Contınuous groups of transformations. Princeton Unıversity Press, 1933.

[8] Eisenhart, L. P., and M. S. Knebelman, Invariant theory of homogeneous contact transformations. Ann. Math. 37 (1936), 747-765.

[9] Gray, J. W., Some global properties of contact structures. Ann. Math. 69 (1959), 421-450.

[10] Hatakeyama, Y., On the existence of Riemannian metrics associated with a 2form of rank $2 r$. Tôhoku Math. J. 14 (1962), 162-166.

[11] Hosokawa, T., Connections in the manifold admitting contact transformations. J. Fac. Sci. Hokkaido Unıv. 2 (1934), 169-176.

[12] Lichnerowicz, A., Théorie globale des connexions et des groupes d'holonomie. Roma, Edizıoni Cremonese, 1955.

[13] Mutō, Y., On the connectıons in the manifold admittıng homogeneous contact transformations. Proc. Phys.-Math. Soc. Japan 20 (1938), 451-457.

[14] Mutō, Y., ANd K. Yano, Sur les transformations de contact et les espaces de Finsler. Tôhoku Math. J. 45 (1939), 293-307.

[15] Rund, H., The differential geometry of Finsler spaces. Berlin, Springer, 1959.

[16] SASAKI, S., Homogeneous contact transformations. Tôhoku Math. J. 14 (1962), 369-397.

[17] Walker, A. G., Connexions for parallel distributions in the large I; II. Quart. J. Math., Oxford (2), 6 (1955), 301-308; 9 (1958), 221-231.

[18] Willmore, T. J., Connexions for systems of parallel distributions. Quart. J. Math., Oxford (2), 7 (1956), 269-276.

[19] Yano, K., On the theory of linear connections in the manifold admitting contact transformations. Proc. Phys.-Math. Soc. Japan 17 (1935), 39-47.

[20] Yano, K., And E. T. Davies, Contact tensor calculus. Ann. Mat. pur. appl. 37 (1954), 1-36.

[21] Yano, K., And E. T. Davies, On the tangent bundles of Finsler and Riemannian manifolds. Rend. Circ. Mat. Palermo (2) 12 (1963), 1-18.

[22] Yano, K., And Y. Mutō, Homogeneous contact structures. Math. Annalen 167 (1966), 195-213.

Department of Mathematics,

Tokyo Institute of Technology and

Department of Applied Mathematics,

Yokoliama National, University. 